\title{
THE SMOOTH CONTINUATION METHOD IN OPTIMAL CONTROL WITH AN APPLICATION TO QUANTUM SYSTEMS
}

\author{
Bernard Bonnard $^{1}$, Nataliya Shcherbakova ${ }^{1}$ and Dominique Sugny ${ }^{2}$
}

\begin{abstract}
The motivation of this article is double. First of all we provide a geometrical framework to the application of the smooth continuation method in optimal control, where the concept of conjugate points is related to the convergence of the method. In particular, it can be applied to the analysis of the global optimality properties of the geodesic flows of a family of Riemannian metrics. Secondly, this study is used to complete the analysis of two-level dissipative quantum systems, where the system is depending upon three physical parameters, which can be used as homotopy parameters, and the timeminimizing trajectory for a prescribed couple of extremities can be analyzed by making a deformation of the Grushin metric on a two-sphere of revolution.
\end{abstract}

Mathematics Subject Classification. 49K15, 65K10, 81V55.

Received April 15, 2009. Revised October 6, 2009.

Published online March 24, 2010.

\section{INTRODUCTION}

A smooth continuation method consists in the following. Suppose one wants to find a solution of a system of equations

$$
F(x)=0,
$$

where $F: \mathbb{R}^{n} \mapsto \mathbb{R}^{n}$ is a smooth mapping. In the smooth continuation method, we consider a smooth homotopy path $H(x, \lambda)$, such that $H(x, 0)=G(x)$ and $H(x, 1)=F(x)$, where $G$ is a map having known zero points. This leads to the smooth numerical continuation method (see [2]), where the solutions are computed iteratively along the path using a Newton-type algorithm. This general method can be applied in Optimal Control. Indeed, from the Pontryagin maximum principle [1,25], the optimal solution for a fixed set of boundary conditions can be computed by solving a shooting equation and the smooth continuation method can be applied if one can construct a smooth homotopy path. In particular, the smooth numerical continuation method was successfully applied to a recent research project in orbital transfer, where low propulsion is used (see for instance $[18,20])$. In such problems, the continuation parameter can be the maximum thrust or it can be introduced as a regularizing parameter in the cost. Indeed, the physical optimal problem amounts to minimize the time of transfer or to maximize the final mass, and in the second case this leads to a $L^{1}$ optimization

\footnotetext{
Keywords and phrases. Optimal control, smooth continuation method, quantum control.

1 Institut de mathématiques de Bourgogne, UMR CNRS 5584, 9 avenue Alain Savary, BP 47870, 21078 Dijon Cedex, France.

2 Institut Carnot de Bourgogne, UMR CNRS 5209, 9 avenue Alain Savary, BP 47870, 21078 Dijon Cedex, France.

Dominique.Sugny@u-bourgogne.fr
} 
problem which is non smooth but can be regularized using an homotopy from $L^{2}$ to $L^{1}$ [19]. This kind of study is interesting from the numerical point of view but leads to very interesting geometric questions, in particular to ensure the convergence of the method. The main problem as pointed out in previous articles (see e.g. [9]) is to relate the smoothness of the path to the concept of conjugate point introduced in [10].

The motivation of this article is double. First of all, our aim is to provide a neat geometric framework for the application of the smooth continuation method in optimal control. To gain smoothness, we restrict to the time-minimum transfer from $q_{0}$ to $q_{1}$ for a system of the form

$$
\frac{\mathrm{d} q}{\mathrm{~d} t}=F_{0}(q)+\sum_{i=1}^{m} u_{i} F_{i}(q)
$$

where the control $u=\left(u_{1}, \ldots, u_{m}\right)$ is such that $\|u\| \leq 1$. Such a problem has many applications in control engineering e.g. in space mechanics (orbit transfer, space mission SMART-1) or in quantum control [16,21] and is a natural generalization of geometric problems: If the drift $F_{0}=0$ and $m=n$, where $n$ is the dimension of the state it corresponds to a Riemannian problem, if $m<n$ it is a sub-Riemannian problem. In the case $F_{0} \neq 0$ it describes the Zermelo-type navigation problem [3]. For all this family of systems the maximum principle leads to the application of a shooting method using the Hamiltonian

$$
H=H_{0}+\left(\sum_{i=1}^{m} H_{i}^{2}\right)^{1 / 2}
$$

where $H_{i}$ denotes the Hamiltonian lift

$$
H_{i}=\left\langle p, F_{i}(q)\right\rangle,
$$

and $H$ is smooth except on the surface $\Sigma: H_{i}=0 ; i=1, \ldots, m$ called the switching surface. To apply the smooth continuation method to this kind of system one must consider a one-parameter family $H_{\lambda}$ of such Hamiltonians. The smoothness of the path and the convergence of the method are related to the non-existence of conjugate points, as introduced in [10] in the smooth case, generalizing the concept of conjugate points for Lagrange problem in the classical calculus of variations [4,24]. Moreover they can be numerically computed using the COTCOT code presented in the afore-mentioned article. Also the variational equation which is used to calculate the conjugate points permits in parallel to generate numerically the derivative of the shooting equation. A striking consequence of this analysis is to deduce the main theoretical result of this article: if we consider a one-parametric family of Riemannian metrics $g_{\lambda}$, a uniform bound of the injectivity radii gives an upper bound between the distance from $q_{0}$ to $q_{1}$ for which the continuation method converges. If this result is an interesting bridge between geometry and convergence of numerical methods, our aim is to illustrate the different points which are important to analyze the more general case: estimation of the position of the conjugate points related to the concept of curvature in the Riemannian case, which has to be numerically computed in general, existence of optimal solutions related to controllability problems, non compactness in general of the domain of the shooting mapping and singularities due to the existence of a switching surface $\Sigma$ on which the Hamiltonian vector field $\vec{H}$ is not smooth. All these problems will be discussed in details in the section devoted to the application of the continuation method to the time-minimal control of two-level dissipative quantum systems which is the second motivation of this article. For such systems, the dynamics is governed by the following equations:

$$
\begin{gathered}
\frac{\mathrm{d} x}{\mathrm{~d} t}=-\Gamma x+u_{2} z \\
\frac{\mathrm{d} y}{\mathrm{~d} t}=-\Gamma y-u_{1} z \\
\frac{\mathrm{d} z}{\mathrm{~d} t}=\gamma_{-}-\gamma_{+} z+u_{1} y-u_{2} x
\end{gathered}
$$


where $\Lambda=\left(\Gamma, \gamma_{-}, \gamma_{+}\right)$is the set of dissipation parameters, and the control $u$ is the complex field $u_{1}+i u_{2}=$ $\|u\| \mathrm{e}^{i \alpha}$, and where up to a rescaling one can assume $\|u\| \leq 1$. There are several reasons to consider that for the time-minimal control problem, the smooth continuation method is well adapted to analyze the system. Indeed, from our previous study $[6,12]$ we know that when $\Gamma=\gamma_{+}$and $\gamma_{-}=0$, the time-minimizing control problem can be reduced to analyze the quasi-Riemannian metric

$$
g=\mathrm{d} \phi^{2}+\tan ^{2} \phi \mathrm{d} \theta^{2}
$$

on the two-sphere of revolution endowed with spherical coordinates $(\phi, \theta)$, called the Grushin metric. For such a metric, the conjugate and cut loci were computed in [11] and it can be taken as a starting point of a continuation method, the one-parameter deformation being either $\lambda=\Gamma-\gamma_{+}$with $\gamma_{-}$fixed or $\lambda=\gamma_{-}$with $\Gamma-\gamma_{+}=0$. Such a study is particularly important in quantum control for the spin $1 / 2$ model, where the control is the magnetic field $[14,21,22,27]$ and the homotopy parameters have a clear physical interpretation. Moreover for these systems, the control is computed as an open-loop function and the continuation method allows to study the dependence of the optimal solution upon dissipation parameters. This is an important issue especially since closed-loop control is delicate to implement in quantum mechanics due to the question of measurements. Also the method can be extended to the $N$-level case, where the dimension of the state space is $N^{2}-1$ [28,29].

This article is organized in two sections. The first section presents the geometric foundations of the smooth continuation method in optimal control. It is based on the previous works in $[5,9,10]$ and the consequence is to produce the global convergence result about the smooth continuation method, in the Riemannian case. Also all the proper concepts are introduced for the application of the method to more general cases, as the one encountered in the quantum control problem. The second section contains the application of this method to the analysis of the time-minimal transfer in two-level dissipative quantum systems. It is composed of three distinct parts. The first one recall the main properties of the extremal flow which is a prerequisite analysis before applying a shooting method. The second part concerns the numerical computation of conjugate points which is crucial to estimate the transfer time for which the continuation method can be applied. In the final part, we present numerical results about the continuation method. We consider homotopies on both $\Gamma-\gamma_{+}$ and $\gamma_{-}$. The continuation method is implemented in a simple numerical framework using Matlab routines, based on a discretization of the homotopy path, and a refined approach which consists in following the smooth path of the zeros solutions by integrating along the path the implicit ODE describing this set [13].

\section{The SMooth CONTINUATion Method in Optimal CONTROL}

\subsection{Preliminaries}

In this section we recall some standard results concerning singular trajectories in optimal control. For a more detailed exposition see [8].

We consider the time optimal control problem with fixed extremities $q_{0}$ and $q_{1}$ for a smooth system

$$
\dot{q}=F(q, u),
$$

where $q \in M, M$ being an $n$-dimensional smooth manifold, and the control function $u(\cdot) \in \mathcal{U}$ where $\mathcal{U}$ is the set of bounded measurable mappings with values in a $m$-dimensional control domain $U$. First of all we recall the maximum principle:

Proposition 2.1. If $(q, u)$ is an optimal control trajectory pair defined on $[0, T]$, then there exists an absolutely continuous trajectory $z=(q, p)$ in $T^{*} M$, such that almost everywhere on $[0, T]$ we have

$$
\dot{q}=\frac{\partial H}{\partial p}(q, p, u), \quad \dot{p}=-\frac{\partial H}{\partial q}(q, p, u)
$$


and

$$
H(q, p, u)=\max _{v \in U} H(q, p, v) .
$$

Moreover, the mapping $z \mapsto M(z)=\max _{v \in U} H(z, v)$ is constant and positive.

Definition 2.1. The mapping

$$
H: \quad T^{*} M \times U \mapsto \mathbb{R}
$$

is called the pseudo-Hamiltonian. A pair $(z, u)$, which solves the maximum principle, is called extremal.

Application 2.1. Assume that $U$ is a $m$-dimensional manifold. Restricting to a chart one can assume that locally $U=\mathbb{R}^{m}$ and the maximization condition leads to

$$
\frac{\partial H}{\partial u}=0
$$

In order to solve this equation we introduce the strong Legendre condition:

(A1) The quadratic form $\frac{\partial^{2} H}{\partial u^{2}}$ is negative definite along the reference trajectory.

According to the implicit function theorem, the extremal control is then locally defined as a smooth function of $z$. Plugging this function into the pseudo-Hamiltonian one gets a smooth true Hamiltonian.

Application 2.2. Consider the control system of the form

$$
F(q, u)=F_{0}(q)+\sum_{i=1}^{m} u_{i} F_{i}(q)
$$

and assume that the control domain is the unit ball $\|u\| \leq 1$. Then the maximization condition leads to the following stratification. Introduce the Hamiltonian lifts

$$
H_{i}(z)=\left\langle p, F_{i}(q)\right\rangle
$$

and let

$$
\Sigma: \quad H_{i}=0, \quad i=1, \ldots, m
$$

be the switching surface. Then outside $\Sigma$ an extremal control is given by

$$
u_{i}=\frac{H_{i}}{\left(\sum_{i=1}^{m} H_{i}^{2}\right)^{1 / 2}}, \quad i=1, \ldots, m .
$$

Plugging this control into $H$ one can define a true Hamiltonian function

$$
H_{r}=H_{0}+\left(\sum_{i=1}^{m} H_{i}^{2}\right)^{1 / 2}
$$

The smooth solutions of the corresponding Hamiltonian system are called extremals of order zero.

The following result is crucial:

Proposition 2.2. Extremal controls of order zero correspond to singularities of the end-point mapping

$$
E^{q_{0}, T}: \quad u(\cdot) \in L^{\infty}[0, T] \mapsto q\left(T, u(\cdot), q_{0}\right)
$$

where $q(\cdot)$ is the trajectory of the system corresponding to $u(\cdot)$ with the control domain on the sphere $\|u\|=1$ and starting at $q_{0}$. 
Proof. The proof is simple and can be obtained by the following computation. For instance, consider the case of $\mathbb{S}^{1}: u_{1}^{2}+u_{2}^{2}=1$. Applying the maximization condition to

$$
H=H_{0}+u_{1} H_{1}+u_{2} H_{2}
$$

outside $\Sigma$ one gets the unique solution (2.1).

If we restrict to $\mathbb{S}^{1}$, we obtain for $\left\|u_{1}\right\| \neq 1$ the condition $u_{2}=\varepsilon \sqrt{1-u_{1}^{2}}, \varepsilon= \pm 1$, and $H$ takes the form

$$
H=H_{0}+u_{1} H_{1}+\varepsilon \sqrt{1-u_{1}^{2}} H_{2} .
$$

Then the maximization condition

$$
\frac{\partial H}{\partial u}=0
$$

gives $H_{1}^{2}=u_{1}^{2}\left(H_{1}^{2}+H_{2}^{2}\right)$. In addition, the Legendre condition $\frac{\partial^{2} H}{\partial u^{2}} \leq 0$ imposes $\varepsilon H_{2} \geq 0$.

As a consequence, we can use all the machinery involving the second order optimality conditions for singular extremals implemented in the COTCOT code. We introduce the following construction (see [8]).

Consider the time minimal control problem for a system of the form $\dot{q}=F(q, u), q(0)=q_{0}$, where $F$ : $\mathbb{R}^{n} \times \mathbb{R}^{m} \mapsto \mathbb{R}^{n}$ is smooth and the control domain is $U=\mathbb{R}^{m}$. Each time-minimal control on $[0, T]$ corresponds to a singularity of the end-point mapping, and from the maximum principle any singular pair $(q, u)$ can be lifted into a singular extremal $(q, p, u)$, where for all $t>0, p$ is orthogonal to the image of the derivative of the end-point mapping evaluated for $\left.u\right|_{[0, t]}$. Assuming (A1) a singular control can be computed as a smooth mapping $u(q, p)$ and we note $H_{r}(z)=H(z, u(z))$. This corresponds precisely to the true Hamiltonian computed for our family of systems

$$
F(q, u)=F_{0}+\sum_{i=1}^{m} u_{i} F_{i}(q), \quad\|u\| \leq 1 .
$$

The extremal is called normal if $H_{r}>0$ and abnormal if $H_{r}=0$. In addition to (A1) we consider the following assumptions:

(A2) $\quad H_{r}>0 \quad$ (normal case).

(A3) The singularity of the end-point mapping is of co-dimension one in each sub-interval of $[0, T]$.

Definition 2.2. Let $\exp t \vec{H}_{r}$ be the one-parameter flow of the Hamiltonian vector field $\vec{H}_{r}$ and $\pi:(q, p) \mapsto q$ be the standard projection. The exponential mapping is the application

$$
\exp _{q_{0}}: \quad \mathbb{R}^{+} \times \mathbb{S}^{n-1} \mapsto \mathbb{R}^{n}
$$

defined by

where $p_{0}$ can be restricted to the unit sphere.

$$
\exp _{q_{0}}\left(t, p_{0}\right)=\pi \exp t \vec{H}_{r}\left(q_{0}, p_{0}\right)
$$

Definition 2.3. As before, let $\vec{H}_{r}(z)$ be the smooth Hamiltonian vector field associated to $H_{r}$, and let $z(t)$ be a reference extremal curve defined on $[0, T]$. The variational equation

$$
\delta \dot{z}=\frac{\partial \vec{H}_{r}}{\partial z}(z) \delta z
$$

is called the Jacobi equation, whose non-trivial solutions $J(t)=(\delta q(t), \delta p(t))$ are called Jacobi fields. The field $J(t)$ is said vertical at time $t$ if $\delta q(t)=0$. A time $t_{c}$ is called (geometrically) conjugate to $t_{0}=0$ if there exists a Jacobi field vertical at zero and at $t_{c}$. In this case the point $q\left(t_{c}\right)$ is said to be conjugate to $q(0)$. 
The next statement is clear.

Proposition 2.3. The point $q\left(t_{c}\right)$ is conjugate to $q(0)$ if and only if the mapping

$$
p_{0} \in \mathbb{S}^{n-1} \mapsto \exp _{q_{0}}\left(t_{c}, p_{0}\right)
$$

is not of full rank $n-1$ at $p_{0}$.

The following result is crucial (see [26]):

Proposition 2.4. Let $z(t)=(q(t), p(t))$ be a reference extremal curve on $[0, T]$ satisfying (A1), (A2) and (A3). The extremal trajectory $q(\cdot)$ is locally time minimal (for the $L^{\infty}$ topology of the sets of control) up to the first geometric conjugate time.

\subsubsection{The algorithm}

Conjugate points are computed numerically using the COTCOT code with the following algorithm.

We restrict to the $(n-1)$-dimensional subspace generated by the Jacobi fields

$$
J_{i}(t)=\left(\delta q_{i}(t), \delta p_{i}(t)\right), \quad i=1, \ldots, n-1
$$

with $\delta q_{i}(0)=0$ and $p(0) \delta p_{i}(0)=0$. A conjugate time occurs at $t=t_{c}$ such that

$$
\operatorname{rank}\left\{M(t)=\mathrm{d} \pi\left(J_{1}(t), \ldots, J_{n-1}(t)\right)\right\} \leq n-2,
$$

where the rank can be computed using a Singular Value Decomposition (SVD) of the matrix $M(t)$, whose smallest singular value vanishes at a conjugate time $t_{c}$.

Since the derivative of the exponential mapping with respect to time $t$ is $\frac{\mathrm{d} q}{\mathrm{~d} t}=F(q, u)$, where $u$ is the extremal control, this is equivalent (in the normal case) to find a zero of the determinant:

$$
\operatorname{det}\left(\delta q_{1}(t), \ldots, \delta q_{n-1}(t), F(q(t), u(t))\right)
$$

This analysis can be extended to the abnormal case for single-input control systems. We next present the geometric framework adapted to our class of systems.

\subsubsection{The bi-input case}

Consider a system of the form

$$
\frac{\mathrm{d} q}{\mathrm{~d} t}=F_{0}(q)+u_{1} F_{1}(q)+u_{2} F_{2}(q), \quad u_{1}^{2}+u_{2}^{2} \leq 1
$$

The corresponding Hamiltonian is given by

$$
H(z)=H_{0}(z)+u_{1} H_{1}(z)+u_{2} H_{2}(z), \quad z \in T^{*} \mathbb{R}^{n} .
$$

In order to describe the extremals for a control on the sphere $u_{1}^{2}+u_{2}^{2}=1$ one can parameterize the controls by setting

$$
u_{1}=\cos \alpha, \quad u_{2}=\sin \alpha
$$

and use $\alpha$ as a new scalar parameter. We get a single input non-linear control system

$$
\frac{\mathrm{d} q}{\mathrm{~d} t}=F_{0}(q)+\cos \alpha F_{1}(q)+\sin \alpha F_{2}(q), \quad \alpha \in \mathbb{R}
$$


On the other hand, by standard Goh transformation one can use as a new control variable the derivative $v=\dot{\alpha}$. Hence the original system is transformed into an extended system, which linearly depends on the control:

$$
\frac{\mathrm{d} \tilde{q}}{\mathrm{~d} t}=\tilde{F}_{0}(\tilde{q})+v \tilde{G}_{0}(\tilde{q}), \quad \tilde{q}=(q, \alpha) \in \mathbb{R}^{n+1} .
$$

The following result is standard.

Proposition 2.5 (see [5]). Generic singular extremals of the system $\left(\tilde{F}_{0}, \tilde{G}_{0}\right)$ and the control

$$
\tilde{u}(\tilde{z})=-\frac{\left\{\left\{H_{\tilde{G}_{0}}, H_{\tilde{F}_{0}}\right\}, H_{\tilde{F}_{0}}\right\}}{\left\{\left\{H_{\tilde{G}_{0}}, H_{\tilde{F}_{0}}\right\}, H_{\tilde{G}_{0}}\right\}}
$$

are the integral curves of a smooth Hamiltonian vector field $\tilde{H}_{r}(\tilde{z})$ of the constrained space

$$
H_{\tilde{G}_{0}}=\left\{H_{\tilde{G}_{0}}, H_{\tilde{F}_{0}}\right\}=0,
$$

where

$$
H_{\tilde{F}_{0}}=\left\langle\tilde{p}, \tilde{F}_{0}\right\rangle, \quad H_{\tilde{G}_{0}}=\left\langle\tilde{p}, \tilde{G}_{0}\right\rangle .
$$

They correspond to singular extremals of the original system.

2.1.3. Algorithm for the computation of $t_{c c}$ in the abnormal case

The following result comes from [5].

Proposition 2.6. Under generic conditions in the abnormal case a singular extremal is time-minimal up to a first conjugate time $t_{c c}$.

Remark 2.1. The conditions for which the result holds are detailed in [5] and can be easily checked.

In the abnormal case the algorithm for computing $t_{c c}$ differs from the one in the normal case. We present it for the extended system. The Jacobi equation is the variational equation associated to $\tilde{H}_{r}(\tilde{z})$. Let us consider the $(n-2)$ Jacobi fields satisfying at time $t=0$ the linearized constraints

$$
\mathrm{d} H_{\tilde{F}_{0}}=\mathrm{d} H_{\tilde{G}_{0}}=\mathrm{d} H_{\left\{\tilde{G}_{0}, \tilde{F}_{0}\right\}}=0
$$

normalized by the condition $\tilde{p}(0) \delta \tilde{p}(0)=0$ and the semi-verticality condition $\delta \tilde{q}(0) \in \mathbb{R} \tilde{G}_{0}\left(\tilde{q}_{0}\right)$. A conjugate time is a time $t_{c c}$ such that

$$
\operatorname{rank}\left\{\mathrm{d} \tilde{\pi}\left(J_{1}\left(t_{c c}\right)\right), \ldots, \mathrm{d} \tilde{\pi}\left(J_{n-2}\left(t_{c c}\right)\right), \tilde{F}_{0}\left(\tilde{q}\left(t_{c c}\right)\right), \tilde{G}_{0}\left(\tilde{q}\left(t_{c c}\right)\right)\right\} \leq n-1 .
$$

It is worth to point out that both normal and abnormal cases can be set in an uniform framework. The Jacobi equations with the corresponding end-points conditions can be reduced to a self-adjoint operator of order $2(n-1)$ in the normal case and of order $2(n-2)$ in the abnormal case, which can be normalized to

$$
D(x)=\left(l_{0} x^{(r)}\right)^{r}+\left(l_{1} x^{(r-1)}\right)^{r-1}+\ldots+l_{r} x=0,
$$

where $r=n-1$ in the normal case and $r=n-2$ in the abnormal one and $l_{0}, \ldots, l_{r}$ are real-valued functions. For instance, if $n=3$, it depends upon three functions $l_{i}(\tilde{q}(t))$ in the normal case and upon two functions in the abnormal case. 


\subsection{Shooting equation, central field and smooth continuation technique}

Definition 2.4. Finding the extremities $q_{0}, q_{1}$, which solve the shooting equation in our problem for $[0, T]$ means solving the equation

$$
E(x)-q_{1}=0
$$

where $x=(p(0), T) \in \mathbb{S}^{n-1} \times \mathbb{R}^{+}$, and $E$ is the exponential mapping $\exp _{q_{0}}$.

The following result is crucial:

Proposition 2.7. In the normal case, the mapping $E$ is of full rank at $x=(p(0), T)$ if and only if $q_{1}$ is not conjugate to $q_{0}$ along the reference extremal.

The shooting equation is connected to the concept of central field that we introduce in the next section.

\subsubsection{Construction of a central field}

Let $z(t)=(q(t), p(t))$ be the reference extremal curve defined on $[0, T]$, i.e., the integral curve of the smooth Hamiltonian vector field satisfying assumptions (A1), (A2) and (A3). In addition we assume that $t \mapsto q(t)$ is one-to-one on $[0, T]$ and $[0, T]$ contains no conjugate time. Therefore by the implicit function theorem we can imbed $q(\cdot)$ into a central field $W$ formed by the extremal curves initiating from $q_{0}$, each point of the domain $W \backslash\left\{q_{0}\right\}$ being the image of a unique pair $\left(p_{1}, t_{1}\right)$ by the exponential mapping. The following proposition is crucial $^{3}$.

Proposition 2.8. Assume that there exists an open neighborhood $W$ of the state space $\mathbb{R}^{n}$ and two smooth mappings $S: W \mapsto \mathbb{R}$ and $\hat{u}: W \mapsto U$ such that for each pair $(q, u)$ we have:

$$
H^{\prime}(q, \mathrm{~d} S(q), \hat{u}(q)) \geq H^{\prime}(q, \mathrm{~d} S(q), u)
$$

where $H^{\prime}(q, p, u)=\langle p, F(q, u)\rangle-1$ and $H^{\prime}(q, \mathrm{~d} S(q), \hat{u}(q))=0$. Then the reference trajectory $t \mapsto q(t)$ with control $u(t)=\hat{u}(q(t))$ is optimal among all the smooth trajectories of the system with the same extremities and contained in $W$.

Proof. Let $0<t_{0}<t_{1} \leq T$ and let $\left(q^{\prime}, u^{\prime}\right)$ be a trajectory of the system on $\left[t_{0}^{\prime}, t_{1}^{\prime}\right]$, contained in $W$ and satisfying the boundary conditions

$$
q\left(t_{0}\right)=q^{\prime}\left(t_{0}^{\prime}\right), \quad q\left(t_{1}\right)=q^{\prime}\left(t_{1}\right) .
$$

If we denote $T(q, \hat{u})=t_{1}-t_{0}$ and $T\left(q, u^{\prime}\right)=t_{1}^{\prime}-t_{0}^{\prime}$ the respective transfer times, then we must prove that $T\left(q^{\prime}, u^{\prime}\right) \geq T(q, \hat{u})$. By definition, we have:

$$
H^{\prime}\left(q^{\prime}(t), \mathrm{d} S\left(q^{\prime}(t)\right), u^{\prime}(t)\right)=\left\langle\mathrm{d} S\left(q^{\prime}(t)\right), F\left(q^{\prime}(t), u^{\prime}(t)\right)\right\rangle-1 .
$$

Therefore integrating we obtain

$$
T\left(q^{\prime}, u^{\prime}\right)=\int_{t_{0}^{\prime}}^{t_{1}^{\prime}} \mathrm{d} t=S\left(q^{\prime}\left(t_{1}^{\prime}\right)\right)-S\left(q^{\prime}\left(t_{0}^{\prime}\right)\right)-\int_{t_{0}^{\prime}}^{t_{1}^{\prime}} H^{\prime}\left(q^{\prime}(t), \mathrm{d} S\left(q^{\prime}(t), u^{\prime}(t)\right)\right) \mathrm{d} t .
$$

Similarly with $H^{\prime}(q(t), d S(q(t)), \hat{u}(q))=0$ we have

$$
T(q, \hat{u})=\int_{t_{0}}^{t_{1}} \mathrm{~d} t=S\left(q\left(t_{1}\right)\right)-S\left(q\left(t_{0}\right)\right) .
$$

\footnotetext{
${ }^{3}$ I. Kupka, private communication.
} 
Hence

$$
T(q, \hat{u})-T\left(q^{\prime}, u^{\prime}\right)=\int_{t_{0}^{\prime}}^{t_{1}^{\prime}} H^{\prime}\left(q^{\prime}(t), \mathrm{d} S\left(q^{\prime}\right), u^{\prime}(t)\right) \mathrm{d} t,
$$

and taking into account the maximization condition we deduce that the integral in the right-hand side is negative.

Construction of $S$. The function $S$ can be found from the Hamilton-Jacobi-Bellman equation

$$
\max _{v \in U} H\left(q, \frac{\partial S}{\partial q}, v\right)=1 .
$$

On the other hand, it can be constructed geometrically using symplectic geometry methods. Let us introduce the set

$$
L=\left\{q, p=\frac{\partial S}{\partial q}\right\}
$$

Then $L$ is a Lagrangian manifold with generating mapping $S$ and moreover, the standard projection $\pi$ : $\left(q, \frac{\partial S}{\partial q}\right) \mapsto q$ is a diffeomorphism. More precisely, we have:

Proposition 2.9. Under our assumptions, the reference extremal curve is time-minimal in a simply connected domain $W$ with respect to all other smooth curves solving the system with the same extremities and contained in the domain covered by the central field.

Proof. The construction is the following. Let $L_{t}$ be the image of the fiber $T_{q_{0}}^{*} M$ by $\exp t \vec{H}_{r}$, and let $L=$ $\bigcup_{t>0} L_{t} \cap\left\{H^{\prime}=0\right\}$. For each $t>0$ the set $L_{t}$ is a Lagrangian manifold, and hence its intersection with the zero-level set $H^{\prime}=0$ is an isotropic manifold of dimension $n-1$. A straightforward computation tells us that $L$ is locally (along the reference extremal curve) a Lagrangian manifold, where $S$ is the generating mapping. The set $W$ is formed by requiring that the standard projection $\pi: L \mapsto M$ is a diffeomorphism.

\subsubsection{Smooth continuation method in the normal case}

In many applications we can imbed the smooth Hamiltonian $H_{r}$ into a smooth family of Hamiltonians $H_{\lambda}(z)$, where $\lambda \in[0,1]$ is a homotopy parameter and $H_{1}(z)=H_{r}$. This leads to a one-parameter family of shooting equations

where

$$
E(x, \lambda)=q_{1},
$$

Considering the normal case, we have the following proposition.

Proposition 2.10. For each $\lambda$ the exponential mapping is of maximal rank if and only if the point $q_{1}$ is nonconjugate to $q_{0}$. Moreover, in this case the solutions of the parameterized shooting equation form a smooth curve, which can be parameterized by $\lambda$.

Proof. The parameterized shooting equation is $E(x, \lambda)=q_{1}$, where $x=(p(0), T)$, and $p(0)$ is normalized by $H_{\lambda}=1$. If $\frac{\partial E}{\partial x}$ is of maximal rank for each $\lambda$, the solution will form a smooth curve $\lambda \mapsto x(\lambda)$.

Hence this leads to a smooth continuation method to compute the solutions of the shooting equation. Obviously this straightforward application of the implicit function has to be adapted to an optimal control problem and more precisely to the concept of central field associated to the problem. The main steps are the following.

1. Starting point of the continuation method. For $\lambda=0$ we pick up a reference extremal $z(t)$ on $\left[0, T_{0}\right]$ starting from $\left(q_{0}, p_{0}\right)$ and satisfying the assumptions. This defines around the reference trajectory a central field with domain $W_{0}$ and $\exp _{q_{0}}\left(p_{0}, T_{0}\right)=q_{1}$. 
Clearly a crucial point is to find the starting point such that $W_{0}$ is the biggest possible in length $T_{0}$ and in size of the conic neighborhood around $p_{0}$.

2. Construction of the path. One must find a discretization $0=\lambda_{0}, \lambda_{1}, \ldots, \lambda_{N}=1$ of $[0,1]$, where the shooting equation is solved iteratively from $\lambda_{i}$ to $\lambda_{i+1}$. If the Newton method is used, this step requires the computation of the derivative of the exponential mapping since

$$
x_{i+1}=x_{i}-E^{\prime}\left(x_{i}\right)^{-1} E\left(x_{i}\right) .
$$

Let us recall the standard result from the differential calculus:

Lemma 2.1. The derivative of the exponential mapping is the solution of the Jacobi equation which is vertical at time $t=0: \delta q(0)=0$.

We then construct a sequence of solutions $\left(p_{0}, T_{0}\right), \ldots,\left(p_{N}, T_{N}\right)$ of the shooting equation, and the corresponding central field domains $W_{0}, \ldots, W_{N}$. In particular, in this construction the crucial point is to ensure the absence of conjugate points in the whole interval $\left[0, T_{i}\right]$. The convergence of the method can also fail because the continuation path can go to the infinity.

Hence practically, a good knowledge of the geometric properties of the extremals is required. We introduce the following definition.

Definition 2.5. Let us denote by $C\left(q_{0}\right)$ the conjugate locus formed by the set of first conjugate points of the extremal curves starting at $q_{0}$. The cut locus $C_{u t}\left(q_{0}\right)$ is the set of points where geodesics cease to be globally optimal. Denote $i\left(q_{0}\right)$ the minimum time to reach the cut locus from $q_{0}$ and the injectivity radius is

$$
i(M, F(q, u), U)=\inf _{q_{0} \in M} i\left(q_{0}\right)
$$

The separating line $S L\left(q_{0}\right)$ is the set where two minimizing geodesics starting at $q_{0}$ intersect.

\subsubsection{An example: the Riemannian case}

In the Riemannian case if we parameterize the curves by arc-length the time minimization problem is equivalent to minimizing the distance. In this case we can prove that the continuation method converges provided that the length of the extremal is shorter than the injectivity radius. More precisely we recall the following classical result [17]:

Proposition 2.11. Let $g$ be a complete Riemannian metric on a manifold $M$. Then

1. A cut point is either a conjugate point or it belongs to the separating line.

2. If $q_{1}$ is a point that realizes the injectivity radius $\inf _{q_{0} \in M} i\left(q_{0}\right)$, then either $q_{1}$ is conjugate to $q_{0}$ or there are two minimizing extremal curves joining $q_{0}$ and $q_{1}$ that form a two-halves of the same closed geodesic.

From this, using the convergence result from [2] we deduce the following theorem.

Theorem 2.1. Let $g_{\lambda}, \lambda \in[0,1]$, be a smooth family of complete Riemannian metrics on $M$. Let us fix the initial point $q_{0}$. Denote $i_{\lambda}\left(q_{0}\right)$ the minimum time the extremal curves starting at $q_{0}$ needs to reach the cut locus, and by $i_{\lambda}=\inf _{q_{0} \in M} i_{\lambda}\left(q_{0}\right)$ the injectivity radius in the corresponding metric. Then

1. For times shorter than $\inf _{\lambda} i_{\lambda}\left(q_{0}\right)$, the continuation method with initial condition of the shooting equation at $q_{0}$ converges.

2. For times shorter than $\inf _{\lambda} i_{\lambda}$ the continuation method converges for every initial condition for the shooting equation. 


\section{Applichtion to the quantum control}

\subsection{Mathematical model}

For dissipative quantum control, the state of an $N$-level system is represented by a density operator $\rho$, which belongs to

$$
\operatorname{pos}(N)=\left\{\rho \in G L(n, \mathbb{C}), \rho={ }^{t} \bar{\rho}, \rho \geq 0, \operatorname{Tr} \rho=1\right\}
$$

and solves the Lindblad equation $[15,16]$

$$
i \dot{\rho}=-\left[H_{u}, \rho\right]+i L(\rho)
$$

where $H_{u}$ is the total Hamiltonian $H_{u}=H_{0}+H \cdot u, H_{0}$ being the field-free Hamiltonian of the system, $H \cdot u=\sum_{i=1}^{m} u_{i} H_{i}$ representing the control field and $L$ being the dissipation operator. More precisely, the equation can be decomposed into the diagonal terms

$$
\dot{\rho}_{n n}=-i\left[H_{u}, \rho\right]_{n n}-\sum_{k \neq n} \gamma_{k n} \rho_{n n}+\gamma_{n k} \rho_{k k}
$$

and off-diagonal terms:

$$
\dot{\rho}_{k n}=-i\left[H_{u}, \rho\right]_{k n}-\Gamma_{k n} \rho_{k n}, \quad k \neq n .
$$

The system depends upon $3(N-1) / 2$ parameters which have the following property: $\gamma_{n k}$ and $\Gamma_{n k}$ are real and non-negative, $\Gamma_{n k}=\Gamma_{k n}$, and moreover, we have certain constraints in form of inequalities to ensure that $\rho \in \operatorname{pos}(N)$, which can be deduced from the Routh-Hurtwitz criteria. For the two-level case controlled by a complex field, making the Rotating Wave Approximation, in suitable coordinates the system takes the following form:

$$
\begin{gathered}
\frac{\mathrm{d} x}{\mathrm{~d} t}=-\Gamma x+u_{2} z \\
\frac{\mathrm{d} y}{\mathrm{~d} t}=-\Gamma y-u_{1} z \\
\frac{\mathrm{d} z}{\mathrm{~d} t}=\gamma_{-}-\gamma_{+} z+u_{1} y-u_{2} x
\end{gathered}
$$

where $\Lambda=\left(\Gamma, \gamma_{+}, \gamma_{-}\right)$is the set of parameters satisfying

$$
2 \Gamma \geq \gamma_{+} \geq\left|\gamma_{-}\right|, \quad \gamma_{+}=\gamma_{12}+\gamma_{21}, \quad \gamma_{-}=\gamma_{12}-\gamma_{21}
$$

and $u=u_{1}+i u_{2}$ is the complex control filed, which can be constrained to $\|u\| \leq 1$, whence $x=2 \operatorname{Re}\left(\rho_{12}\right)$, $y=2 \operatorname{Im}\left(\rho_{12}\right)$ and $z=\rho_{22}-\rho_{11}$. For this system the unit ball $\|q\| \leq 1$ is invariant for the dynamics, while $\|q\|=1$ represents pure states. In this form, the parameters are related to those in Bloch equation, $\Gamma$ and $\gamma_{+}$are the rates of decreasing of $x$ and $z$ respectively, while $\gamma_{-} / \gamma_{+}$represents the equilibrium state of the free motion.

In this representation, the dissipation corresponds to the drift term, while controls $u_{1}$ and $u_{2}$ act as rotation around $x$ and $y$ axes respectively. Next we introduce the geometric coordinates to analyze the time-minimal control problem.

\subsection{Geometric coordinates}

To make the geometric analysis, the system is written using the spherical coordinates $(\rho, \theta, \phi)$, where $\rho$ is the distance to the origin which represents the purity of the system (a pure state is a point of the sphere), $\theta$ is the angle around the $z$-axis, and $\phi$ is the angle along the meridian from the North pole. Setting

$$
x=\rho \sin \phi \cos \theta, \quad y=\rho \sin \phi \sin \theta, \quad z=\rho \cos \phi,
$$


and making a feedback transformation

$$
v_{1}=u_{1} \cos \theta+u_{2} \sin \theta, \quad v_{2}=-u_{1} \sin \theta+u_{2} \cos \theta
$$

we obtain the following system:

$$
\frac{\mathrm{d} \rho}{\mathrm{d} t}=\gamma_{-} \cos \phi-\rho\left(\gamma_{+} \cos ^{2} \phi+\Gamma \sin ^{2} \phi\right)
$$

and

$$
\begin{gathered}
\frac{\mathrm{d} \phi}{\mathrm{d} t}=-\frac{\gamma_{-} \sin \phi}{\rho}+\frac{\sin 2 \phi}{2}\left(\gamma_{+}-\Gamma\right)+v_{2} \\
\frac{\mathrm{d} \theta}{\mathrm{d} t}=-v_{1} \cot \phi .
\end{gathered}
$$

It represents a coupling between the evolution of the purity and the system on the two-sphere of revolution. This leads to the definition of the geometric problem, where only the $\phi$ and $\theta$ variables are controlled. This corresponds to the so-called reduced problem. In addition, the energy

$$
\int_{0}^{T}\left(v_{1}^{2}+v_{2}^{2}\right) \mathrm{d} t
$$

defines a metric $g=\mathrm{d} \phi^{2}+\tan ^{2} \mathrm{~d} \theta^{2}$ on the two-sphere of revolution. This metric is called the standard Grushin metric on the two-sphere of revolution.

The Hamiltonian $H_{r}$, which corresponds to the extremals of order zero, in spherical coordinates takes the form

$$
H_{r}=-\rho\left(\gamma_{+} \cos ^{2} \phi+\Gamma \sin ^{2} \phi\right) p_{\rho}+\left[-\frac{\gamma_{-} \sin \phi}{\rho}+\frac{1}{2} \sin 2 \phi\left(\gamma_{+}-\Gamma\right)\right] p_{\phi}+\sqrt{p_{\phi}^{2}+p_{\theta}^{2} \cot ^{2} \phi}
$$

Notice that $H_{r}$ does not depend on $\theta$, so we can immediately deduce that $p_{\theta}$ is an integral of the motion. Moreover, we have:

Proposition 3.1. Let $r=\ln \rho$ be a new variable. Then for $\gamma_{-}=0$ the Hamiltonian reads

$$
H_{r}=-\left(\gamma_{+} \cos ^{2} \phi+\Gamma \sin ^{2} \phi\right) p_{r}+\frac{1}{2} \sin 2 \phi\left(\gamma_{+}-\Gamma\right) p_{\phi}+\sqrt{p_{\phi}^{2}+p_{\theta}^{2} \cot ^{2} \phi}
$$

Hence $r$ and $\theta$ are cyclic coordinates and $p_{r}$ and $p_{\theta}$ are first integrals of the motion. Thus the system in this case is Liouville integrable.

\subsection{Parameterization of the extremals in the integrable case}

For the analysis of the problem by means of a continuation method it is important to have an explicit representation of the extremals in the integrable case. Also in this case the variational equation is integrable and in particular one can compute the Jacobian of the shooting equation.

\subsubsection{The Grushin case}

In this case the Hamiltonian takes the form

$$
H_{r}=\frac{1}{2}\left(p_{\phi}^{2}+\frac{p_{\theta}^{2}}{G(\phi)}\right), \quad G(\phi)=\tan ^{2} \phi .
$$


If we introduce $G_{\lambda}(\phi)=\frac{\sin ^{2} \phi}{1-\lambda \sin ^{\phi}}$, where $\lambda \in[0,1]$, then the one-parameter family of Hamiltonians

$$
H_{\lambda}=\frac{1}{2}\left(p_{\phi}^{2}+\frac{p_{\theta}^{2}}{G_{\lambda}(\phi)}\right)
$$

shows that we have an homotopy from the round metric on the two-sphere of revolution, which corresponds to $\lambda=0$, to the Grushin case with $\lambda=1$, so that for $\lambda \in[0,1[$ the associated family of metrics are Riemannian metrics on the whole sphere, while for $\lambda=1$ we have a pole for the metric on the equator $\phi=\pi / 2$. This singularity has no consequence for the parameterized extremals. Using $\frac{\mathrm{d} \phi}{\mathrm{d} t}=p_{\phi}$, one can write

$$
H_{\lambda}=H_{1}+\frac{1}{2} p_{\theta}^{2}(1-\lambda)
$$

and parameterizing by arc-length: $H_{\lambda}=1 / 2$, one gets the level set

$$
H_{1}=\frac{1}{2}-\frac{1}{2} p_{\theta}^{2}(1-\lambda)
$$

Hence the integration of the Grushin case gives the solution for every $\lambda$ and the extremals have the same transcendence. In the Grushin case, the integration is as follows.

We write

$$
H_{1}=\frac{1}{2}\left[\left(\frac{\mathrm{d} \phi}{\mathrm{d} r}\right)^{2}+\nu \cot ^{2} \phi\right], \quad \nu=p_{\theta}^{2}>0,
$$

and we fix the level set to $1 / 2$. To integrate, we observe that we can consider the trajectory starting at $t=0$ from the equator. Consider the branch in the North hemisphere such that $\frac{\mathrm{d} \phi}{\mathrm{d} t}<0$. Let us introduce the angle $\psi=\pi / 2-\phi$. Then we have

$$
\int \frac{\cos \psi \mathrm{d} \psi}{\left(1-m^{2} \sin ^{2} \psi\right)^{1 / 2}}=\frac{1}{m} \arcsin (m \sin \psi),
$$

where $m^{2}=\nu+1$. Hence we obtain

$$
\phi(t)=-\arcsin \left[\frac{1}{m} \sin m t\right]+\frac{\pi}{2}
$$

To complete the integration, we write

$$
\frac{\mathrm{d} \theta}{\mathrm{d} t}=\frac{p_{\theta}}{\sin ^{2} \phi}-p_{\theta}
$$

and use the formula

$$
\int \frac{\mathrm{d} x}{1-a \sin ^{2} x}=\frac{1}{\sqrt{1-a}} \arctan [\sqrt{1-a} \tan x]
$$

for $a<1$. This leads to the following proposition.

Proposition 3.2. The branches of extremals in the North hemisphere starting from the equator, not contained in a meridian plane and such that $\phi$ is decreasing, are given by

$$
\begin{gathered}
\phi(t)=\frac{\pi}{2}-\arcsin \left[\frac{1}{m} \sin m t\right], \quad m=\left(p_{\theta}^{2}+1\right)^{1 / 2}, \\
\theta(t)=p_{\theta}\left[\frac{1}{\sqrt{m^{2}-1}} \arctan \frac{\sqrt{m^{2}-1}}{m} \tan t-t\right] .
\end{gathered}
$$




\subsubsection{Integrability and homotopy}

In the Riemannian case we can either minimize the time, parameterizing the trajectories by arc-length, or minimize the energy. For a system with a drift this leads to two different Hamiltonians, which can be related through the homotopy

$$
H_{\lambda}=H_{0}+\left(H_{1}^{2}+H_{2}^{2}\right)^{\lambda}
$$

where $\lambda=1 / 2$ corresponds to the time and $\lambda=1$ corresponds to the energy minimizing problems.

One can also consider the imbedding into a 3-dimensional Riemannian problem

$$
H_{\lambda}=\left(\lambda H_{0}\right)^{2}+H_{1}^{2}+H_{2}^{2}
$$

where $\lambda$ is a weight ponderating the drift. The extremal trajectories can be tracked by the true system, if the corresponding component $u_{0}=\lambda H_{0}$ is positive.

Hence, besides the parameters in the model which can be used to define homotopy paths, we can introduce homotopy parameters related to homotopy in the cost. Also we can replace the time-minimal control by the problem of calculating the solution which represents an extremum of the purity $\rho$ of the system. This problem is connected to the problem of minimizing time but with no end-point condition on this variable, this is the so-called reduced problem. All those problems can be described by a family of Hamiltonians depending upon parameters, in which the Hamiltonian problem associated to time is imbedded. With respect to those Hamiltonians, the Grushin one represents the candidate to start the continuation, especially if there is no end-point condition on the purity.

Next we discuss the energy minimization problem since in this case one can get an explicit parameterization of the Hamiltonian if $\gamma_{-}=0$.

\subsubsection{The case of energy}

In the normal case, if $\gamma_{-}=0$ the Hamiltonian is

$$
H=-p_{r}\left(\gamma_{+} \cos ^{2} \phi+\Gamma \sin ^{2} \phi\right)+\frac{1}{2} p_{\phi} \sin 2 \phi\left(\gamma_{+}-\Gamma\right)+\frac{1}{2}\left(p_{\phi}^{2}+p_{\theta}^{2} \cot ^{2} \phi\right) .
$$

One has

$$
p_{\phi}=\frac{\mathrm{d} \phi}{\mathrm{d} t}+\frac{\Gamma-\gamma_{+}}{2} \sin 2 \phi
$$

If we fix the level set to $h$, then we get

$$
\frac{1}{2}\left(\frac{\mathrm{d} \phi}{\mathrm{d} t}\right)^{2}+V(\phi)=h
$$

where $V(\phi)$ represents a potential given by

$$
V(\phi)=-p_{r}\left(\gamma_{+} \cos ^{2} \phi+\Gamma \sin ^{2} \phi\right)-\frac{1}{2} \frac{\left(\Gamma-\gamma_{+}\right)^{2}}{4} \sin ^{2} 2 \phi+\frac{1}{2} p_{\theta}^{2} \cot ^{2} \phi,
$$

which describes the evolution of the $\phi$-variable associated to a mechanical system. In particular, the solutions can be understood by analyzing the graph of $V(\phi)$, and the equilibrium states can be obtained by solving $\frac{\partial V}{\partial \phi}=0$. A trivial solution corresponds to the equator $\phi=\pi / 2$, otherwise we must solve

$$
2 p_{r}\left(\Gamma-\gamma_{+}\right) \sin ^{4} \phi+\sin ^{4} \phi\left(1-2 \sin ^{2} \phi\right)\left(\Gamma-\gamma_{+}\right)^{2}+p_{\theta}^{2}=0
$$

To find the solution from $\frac{\mathrm{d} \phi}{\mathrm{d} t}= \pm \sqrt{2(h-V(\phi)}$, we must evaluate an elliptic integral of the form

$$
\int \frac{\mathrm{d} \phi}{\sqrt{2(h-V(\phi))}}=\int \frac{\mathrm{d} X}{\sqrt{P(X)}}
$$


where $X=\sin ^{2} \phi$ and

$$
P(X)=4(1-X)\left[-X^{3}\left(\Gamma-\gamma_{+}\right)^{2}+X^{2}\left(\Gamma-\gamma_{+}\right)\left(2 p_{r}+\Gamma-\gamma_{+}\right)+X\left(2 h+2 p_{r} \gamma_{+}+p_{\theta}^{2}\right)-p_{\theta}^{2}\right] .
$$

This calculation can be reduced to an elliptic integral of the first kind using a transformation of the form (see [23]):

$$
u=\frac{X-p}{X-q}
$$

Hence $X$ is the image of the inverse transformation of an elliptic function of the first kind. To integrate the remaining variable, we write

$$
\frac{\mathrm{d} \theta}{\mathrm{d} t}=p_{\theta}\left(\frac{1}{X}-1\right), \quad \frac{\mathrm{d} r}{\mathrm{~d} t}=-\left(\Gamma-\gamma_{+}\right) X-\gamma_{+} .
$$

These equations can be integrated using special functions according to formulae of [23], p. 92.

The time minimal case is more intricate because we cannot reduce the problem to the integration of a one-dimensional mechanical system.

\subsection{Classification of the asymptotics of the extremal solutions}

This classification coming from [12] is important because it is related to the accessibility problem and to the existence of conjugate points. The extremal curves of order zero are solutions of

$$
\begin{gathered}
\dot{\rho}=\gamma_{-} \cos \phi-\rho\left(\gamma_{+} \cos ^{2} \phi+\Gamma \sin ^{2} \phi\right), \\
\dot{\phi}=\frac{\gamma_{-} \sin \phi}{\rho}+\frac{\gamma_{+}-\Gamma}{2} \sin 2 \phi+\frac{p_{\phi}}{Q} \\
\dot{\theta}=\frac{p_{\theta} \cot ^{2} \phi}{Q}
\end{gathered}
$$

and

$$
\begin{gathered}
\dot{p}_{\rho}=\left(\gamma_{+} \cos ^{2} \phi+\Gamma \sin ^{2} \phi\right) p_{\rho}-\frac{\gamma_{-}}{\rho^{2}} \sin \phi p_{\phi}, \\
\dot{p}_{\phi}=\left(\gamma_{-} \sin \phi+\rho\left(\Gamma-\gamma_{+}\right) \sin 2 \phi\right) p_{\rho}-\left(-\frac{\gamma_{-}}{\rho} \cos \phi+\left(\gamma_{+}-\Gamma\right) \cos 2 \phi\right) p_{\phi}+\frac{\cos \phi}{Q \sin ^{3} \phi} p_{\theta}^{2}, \\
\dot{p}_{\theta}=0, \quad Q=\sqrt{p_{\theta}^{2} \cot ^{2} \phi+p_{\phi}^{2}} .
\end{gathered}
$$

The behaviors of the solutions are intricate, but one can classify them by their asymptotic properties. First of all, let us describe the solutions in the Grushin case.

\subsubsection{Classification of the extremals in the Grushin case}

Assume $\gamma_{-}=0, \Gamma=\gamma_{+}$. In this case the purity $\rho$ of the system cannot be controlled and the study of the extremals reduces to the analysis of the geodesics of the Grushin metric on the two sphere of revolution, which was done in [6]. The geodesics are

a) meridian circles

or

b) periodic trajectories in the plane $\left(\phi, p_{\phi}\right)$ with $\dot{\theta}$ periodic. Moreover, these trajectories are invariant for the central symmetry: $\left(\phi, p_{\phi}\right) \mapsto\left(\pi-\phi,-p_{\phi}\right)$, and can be constructed by integrating over a half-period. 


\subsubsection{Classification of extremals in the case $\gamma_{-}=0$}

In this case, on the two-sphere of revolution, the reduced system defines a system of the form

$$
\dot{q}=G_{0}(q)+\sum_{i=1}^{2} u_{i} G_{i}(q) .
$$

It describes a Zermelo navigation problem on the two-sphere, where the drift term $G_{0}$ represents the current:

$$
G_{0}=\frac{\gamma_{+}-\Gamma}{2} \sin 2 \phi \frac{\partial}{\partial \rho}
$$

and

$$
G_{1}=\frac{\partial}{\partial \phi}, \quad G_{2}=-\cot \phi \frac{\partial}{\partial \theta}
$$

form a frame for the metric $g=d \phi^{2}+\tan ^{2} \mathrm{~d} \theta^{2}$, which is singular on the equator $\phi=\pi / 2$. The current can be compensated by a feedback with $\|u\|<1$ if $\left|\gamma_{+}-\Gamma\right|<2$. This leads to the following discussion.

Case $\left|\gamma_{+}-\Gamma\right|<2$. In this case the reduced system defines a Finsler geometric problem for which roughly the extremals are deformations of the geodesics of $g=\mathrm{d} \phi^{2}+\tan ^{2} \mathrm{~d} \theta^{2}$. The main properties are described below.

Proposition 3.3. If for fixed $\left(p_{r}, p_{\theta}\right)$ the level set of $H_{r}=\varepsilon, \varepsilon \in\{0,1\}$, is compact, contains no singular points and admits a central symmetry with respect to the set $\left\{\phi=\pi / 2, p_{\phi}=0\right\}$, then it contains a periodic trajectory $\left(\phi, p_{\phi}\right)$ of period $T$ and we have two generically distinct extremals curves $q^{+}(t), q^{-}(t)$, starting from the same point and intersecting with the same length $T / 2$ at a point such that $\phi(T / 2)=\pi-\phi(0)$.

Only these extremals are observed numerically.

Case $\left|\gamma_{+}-\Gamma\right|>2$. We have two types of extremals, characterized by their projection on the two-sphere: Those lying in a band along the equator and described by the previous proposition, and those crossing the band near $\phi=\pi / 4$, and whose asymptotic properties are described in the next proposition.

Proposition 3.4. If $\left|\Gamma-\gamma_{+}\right| \geq 2$, then we have extremal trajectories such that $\dot{\phi} \rightarrow 0,\left|p_{\phi}\right| \rightarrow+\infty$ when $t \rightarrow+\infty$, while $\dot{\theta} \rightarrow 0$.

Proof. The existence of such extremals is related to the non-compactness of the level set of the Hamiltonian. Their appearance becomes clear from the following geometric property. In the North hemisphere the current is maximal for $\phi_{0}=\pi / 4$, and it cannot be compensated in a band $\left|\phi-\phi_{0}\right| \leq M$. Indeed, consider the reduced system on the sphere. By taking $v_{1}=0$ we restrict the problem to meridian planes. Then the system becomes

$$
\frac{\mathrm{d} \phi}{\mathrm{d} t}=\frac{\gamma_{+}-\Gamma}{2} \sin 2 \phi+v_{2}
$$

Taking $v_{2}$ with magnitude 1 , this equation has a singularity when

$$
\frac{\left(\gamma_{+}-\Gamma\right)^{2}}{4} \sin ^{2} 2 \phi=1
$$

This defines the asymptotic set $S$ for the $\phi$ variable.

The integrable case is non-generic, but it guides the classification of the generic case. 
3.4.3. Generic classification of the extremals in the case $\gamma_{-} \neq 0$

We have two cases, observed in the numerical calculations.

Case a). It is a situation where $\left|p_{\phi}\right| \rightarrow+\infty$ as $t \rightarrow+\infty$, while $\cot \phi$ remains finite. Hence $v_{1} \rightarrow 0$ and $v \rightarrow \varepsilon= \pm 1$. From the system we deduce the relations:

$$
\begin{gathered}
\frac{\gamma_{-}}{\rho} \cos \phi=\gamma_{+} \cos ^{2} \phi+\Gamma \sin ^{2} \phi \\
\frac{\gamma_{-}}{\rho} \sin \phi=\left(\gamma_{+}-\Gamma\right) \cos \phi \sin \phi+\varepsilon .
\end{gathered}
$$

Taking the quotient and simplifying one gets that $\tan \phi=\varepsilon / \Gamma$, which gives the asymptotics:

$$
\phi=\arctan \frac{1}{\Gamma} \quad \text { if } \quad \gamma_{-}>0
$$

or

$$
\phi=\pi-\arctan \frac{1}{\Gamma} \quad \text { if } \quad \gamma_{-}<0 .
$$

From that, we deduce the asymptotic for $\rho$. We have:

Proposition 3.5. In the case a), where $\left|p_{\phi}(t)\right| \rightarrow+\infty$ as $t \rightarrow+\infty$, the asymptotic stationary points $\left(\rho_{F}, \phi_{F}, \theta_{F}\right)$ of the dynamics are given by

$$
\begin{gathered}
\rho_{F}=\frac{\left|\gamma_{-}\right| \sqrt{1+\Gamma^{2}}}{1+\gamma_{+} \Gamma}, \\
\phi_{F}=\left\{\begin{array}{ccc}
\arctan \frac{1}{\Gamma} & \text { if } & \gamma_{-}>0 \\
\pi-\arctan \frac{1}{\Gamma} & \text { if } & \gamma_{-}<0 .
\end{array}\right.
\end{gathered}
$$

Case $\mathbf{b})$. It is the situation where $p_{\phi}$ is unbounded but oscillates while $\phi \rightarrow 0(\bmod \pi)$ as $t \rightarrow+\infty$ and at the infinity $p_{\phi}$ dominates $\cot \phi$. The control field $v_{2}$ acquires here a bang-bang structure related to the unbounded and oscillating behavior of $p_{\phi}$. In this case $\phi$ tends to 0 or $\pi$ and the limit is not depending upon the parameters. The asymptotic of $\rho$ can be easily computed.

Proposition 3.6. In the case $\mathrm{b})$, where $\lim _{t \rightarrow+\infty} \phi(t)=0 \bmod \pi$, the asymptotic limit of the dynamics is characterized by

$$
\begin{gathered}
\rho_{F}=\frac{\left|\gamma_{-}\right|}{\gamma_{+}}, \\
\phi_{F}=\left\{\begin{array}{lll}
0 & \text { if } & \gamma_{-}>0 \\
\pi & \text { if } & \gamma_{-}<0
\end{array}\right.
\end{gathered}
$$

\subsection{Numerical estimation of the conjugate points}

The estimation of conjugate and cut points is important for the convergence of the continuation method. We present the results using numerical simulations.

\subsubsection{The Grushin case}

In this case we can compute the conjugate and cut points following the results of [11]. Indeed, in the Grushin case the $\rho$ variable cannot be controlled and we can restrict the analysis to the Grushin metric $g=\mathrm{d} \phi^{2}+\tan ^{2} \phi \mathrm{d} \theta^{2}$ on the two sphere of revolution. For the computation of the conjugate and cut loci of a point $q_{0}=(\phi(0), \theta(0))$, where $\theta(0)$ can be set to 0 , we distinguish the following three cases.

- $\phi(0)=0$ or $\pi$. In this case $q_{0}$ is a pole and the conjugate and cut loci are formed by the antipodal pole; 
- $\phi(0) \neq \frac{k \pi}{2}, k=0,1, \ldots$ If $q_{0}$ is neither a pole nor a point on the equator, the cut locus is a segment of the antipodal parallel, while the conjugate locus has the shape of an astroid with four cusps. The distance to the cut locus can be easily computed: it is the length of the unique geodesic starting tangentially to the parallel and reaching the antipodal parallel in a tangent way.

- $\phi(0)=\pi / 2$. In this case the cut locus is the whole equator minus the initial point, while the conjugate points accumulate at $\phi(0)$. Hence the distance to the cut locus is zero.

In conclusion, we have an exact computation of the distance to the cut locus. But since the conjugate points accumulate near the initial point when $\phi(0)=\pi / 2$, the injectivity radius of the metric is zero.

We present numerical estimates of the first conjugate point both in the abnormal and normal cases. It is motivated by the following observation.

Lemma 3.1. In the Grushin case a singular trajectory is not strict, i.e., it admits a normal and an abnormal extremal lift.

Proof. Indeed, the co-dimension of the singularity mapping is clearly two, since $\rho$ is not controllable. Taking the coordinates $(r, \phi, \theta)$, the adjoint component can be set to 0 or to 1 in the Hamiltonian $H_{r}$ reduced to $\Gamma p_{r}+\sqrt{p_{\phi}^{2}+\cot ^{2} \phi p_{\theta}^{2}}$. In the first case $H_{r} \neq 0$, but in the second case $H_{r}$ can be set to 0 , which corresponds to the abnormal case.

\subsubsection{Estimation of the conjugate points in the abnormal case}

In this case, the abnormal extremal flow can be computed as a system

$$
\frac{\mathrm{d} \tilde{q}}{\mathrm{~d} t}=\tilde{F}(\tilde{q})+v \tilde{G}(\tilde{q})
$$

in dimension 4 , where $\tilde{q}=(x, y, z, v)$. We use the following relations:

$$
\begin{gathered}
\langle\tilde{p}, \tilde{F}\rangle=\langle\tilde{p}, \tilde{G}\rangle=\langle\tilde{p},[\tilde{G}, \tilde{F}]\rangle=0, \\
\langle\tilde{p},[[\tilde{G}, \tilde{F}], \tilde{F}]+v[[\tilde{G}, \tilde{F}], \tilde{G}]\rangle=0 .
\end{gathered}
$$

Eliminating $\tilde{p}$, the abnormal control can be found from the equation

$$
D+v_{s} D^{\prime}=0
$$

where we have introduced the determinants

$$
\begin{aligned}
D & =\operatorname{det}\{\tilde{F}, \tilde{G},[\tilde{G}, \tilde{F}],[[\tilde{G}, \tilde{F}], \tilde{F}]\}, \\
D^{\prime} & =\operatorname{det}\{\tilde{F}, \tilde{G},[\tilde{G}, \tilde{F}],[[\tilde{G}, \tilde{F}], \tilde{G}]\},
\end{aligned}
$$

and the abnormal extremal flow is defined by a vector field denoted $\tilde{F}_{s}$, while the variational equation reduces to

$$
\frac{\mathrm{d} \delta \tilde{q}}{\mathrm{~d} t}=\frac{\partial \tilde{F}_{s}}{\partial q}(\tilde{q}(t)) \delta \tilde{q} .
$$

To test the existence of conjugate points, one has to compute the single Jacobi field $J_{1}(t)$, whose projection on the $\tilde{q}$-state is denoted by $\delta_{1} \tilde{q}$ and is obtained by integrating the variational equation with the initial condition

$$
\delta_{1} \tilde{q}(0)=\frac{\partial}{\partial \alpha}
$$



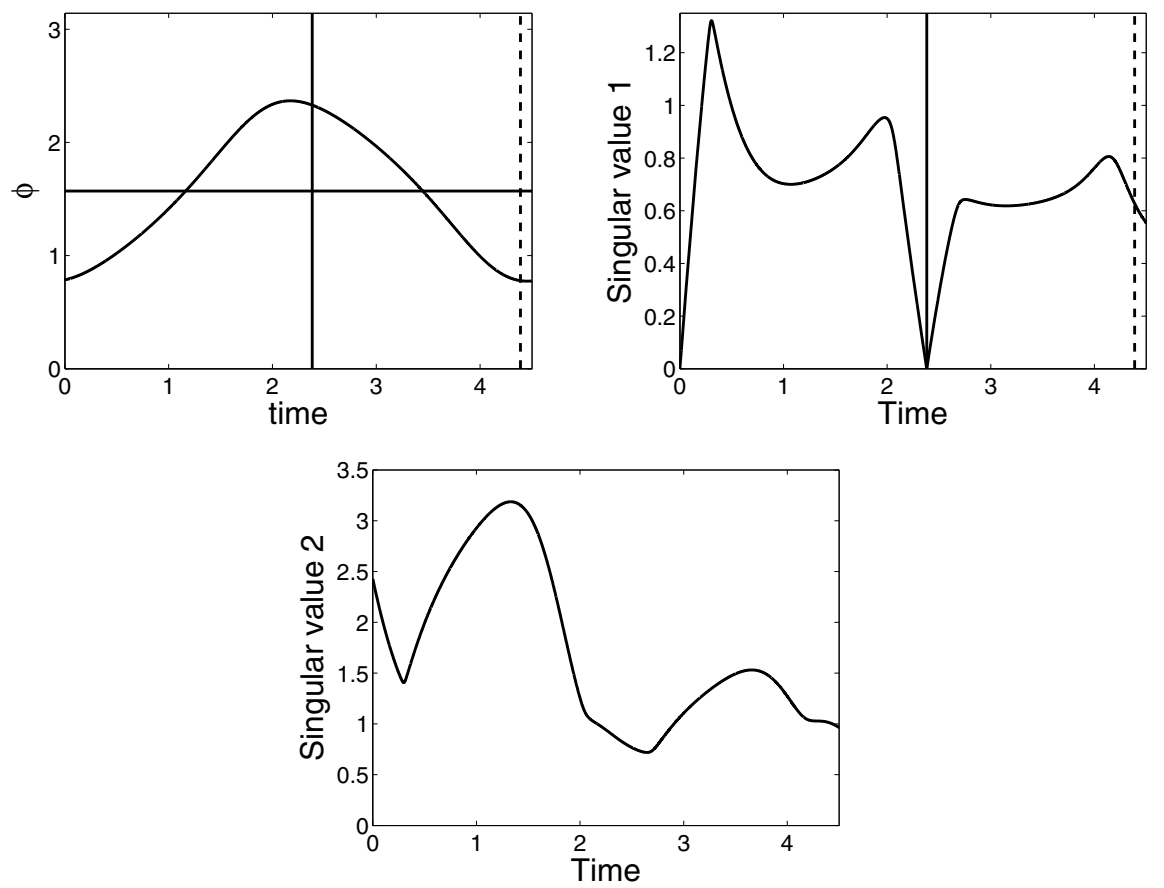

Figure 1. Evolution of the angle $\phi$ in the abnormal case as a function of time for $\Gamma=2.5$, $\gamma_{+}=2$ and $\gamma_{-}=0$. Initial values are taken to be $\phi(0)=\pi / 4, p_{\theta}=2$ and $p_{\phi}(0)=1$. The horizontal solid line indicates the position of the equator. Evolution of the two singular values as a function of time. The solid and dashed vertical lines correspond respectively to the position of the conjugate point with the abnormal (time $t_{c c}$ ) and normal tests (time $t_{c}$ ).

The conjugate point calculation concludes by checking the semi-verticality test

$$
\delta_{1} \tilde{q}\left(t_{c c}\right) \in \operatorname{span}\left\{\tilde{G}\left(\tilde{q}\left(t_{c c}\right)\right), \tilde{F}\left(\tilde{q}\left(t_{c c}\right)\right)\right\} .
$$

This corresponds to the optimality test provided the generic conditions described in [5] are satisfied.

This test can be also checked on the original system in the form $\delta_{1} q\left(t_{c c}\right) \in \mathbb{R}\left(\dot{q}\left(t_{c c}\right)\right)$. Computing the Lie brackets one gets:

$$
\begin{gathered}
D^{\prime}=z\left(-\Gamma\left(x^{2}+y^{2}\right)+\gamma_{-} z-\gamma_{+} z^{2}\right), \\
D=(x \cos \alpha+y \sin \alpha)\left[z^{2}\left(2 \Gamma-\gamma_{+}\right)+(x \sin \alpha-y \cos \alpha)\left(2 \Gamma z\left(\gamma_{+}-\Gamma \gamma_{-}\right)-\Gamma \gamma_{-}\right)+\Gamma\left(x^{2}+y^{2}\right)\right] .
\end{gathered}
$$

The result of the simulations using a SVD to test the conjugate points for the original system are represented below in Figures 1 and 2 for $\gamma_{-}=0$ and $\gamma_{-} \neq 0$. We have chosen examples where conjugate points occur. Another information of these figures is the location of conjugate points computed with the test in the normal case, which confirms numerically the theoretical result $t_{c c} \leq t_{c}$.

\subsubsection{Estimation of the conjugate points in the normal case}

Since the system is of dimension 4 , one needs to compute two Jacobi fields to find the conjugate points. We present in Figures 3-6 the results of the test using a SVD technique. Also we observe the following:

- In the integrable case $\gamma_{-}=0$ conjugate points occur only when the evolution of the $\phi$ variable is periodic as can be seen in Figure 4. We observe no conjugate point in Figure 3 where $\phi$ tends to a constant as $t \rightarrow+\infty$. 

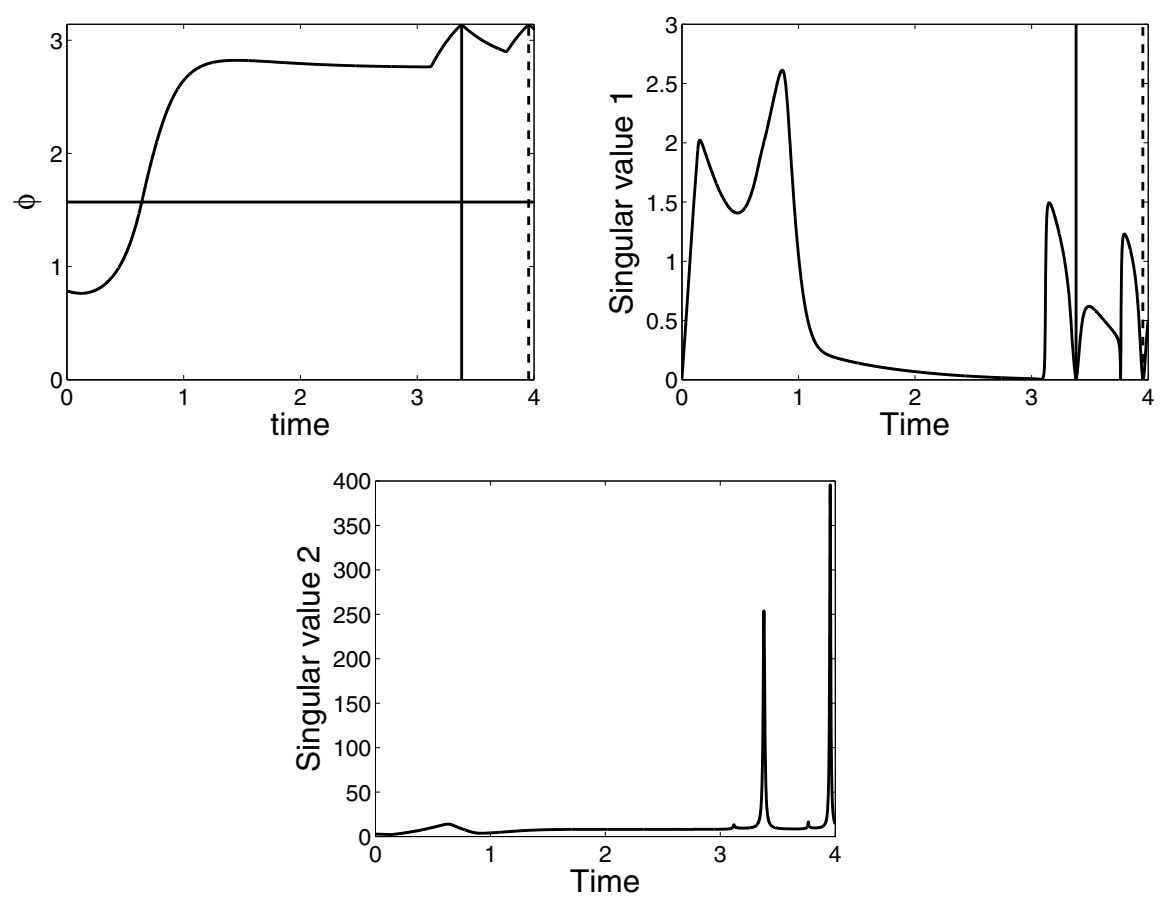

Figure 2. Same as Figure 1 but for $p_{\phi}(0)=-1, \Gamma=2.5$ and $\gamma_{-}=-0.5$.
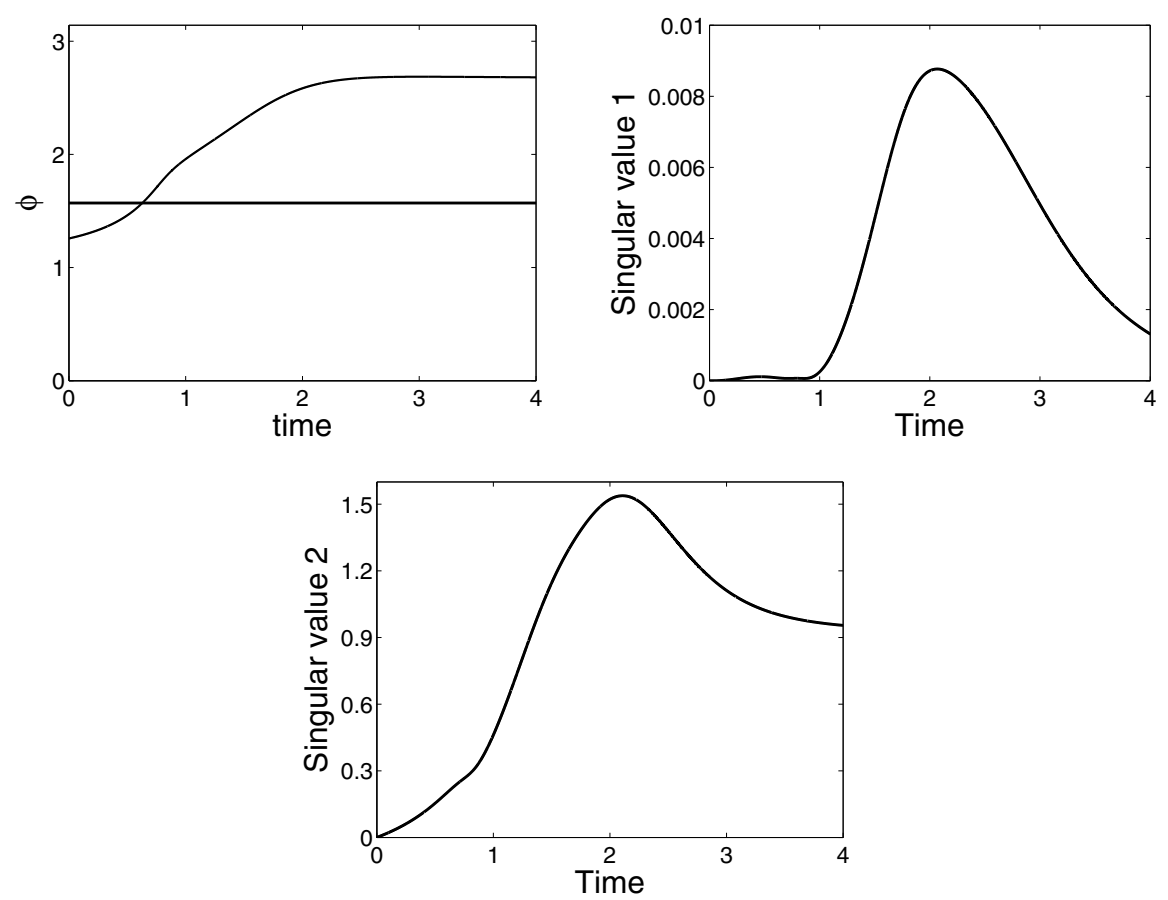

Figure 3. Evolution of the angle $\phi$ as a function of time for $\Gamma=4.5, \gamma_{+}=2$ and $\gamma_{-}=0$. Initial values are taken to be $\phi(0)=2 \pi / 5, p_{\rho}(0)=0.25, p_{\theta}=8$ and $p_{\phi}(0)=10$. Evolution of the two singular values as a function of time. We observe no conjugate point. 

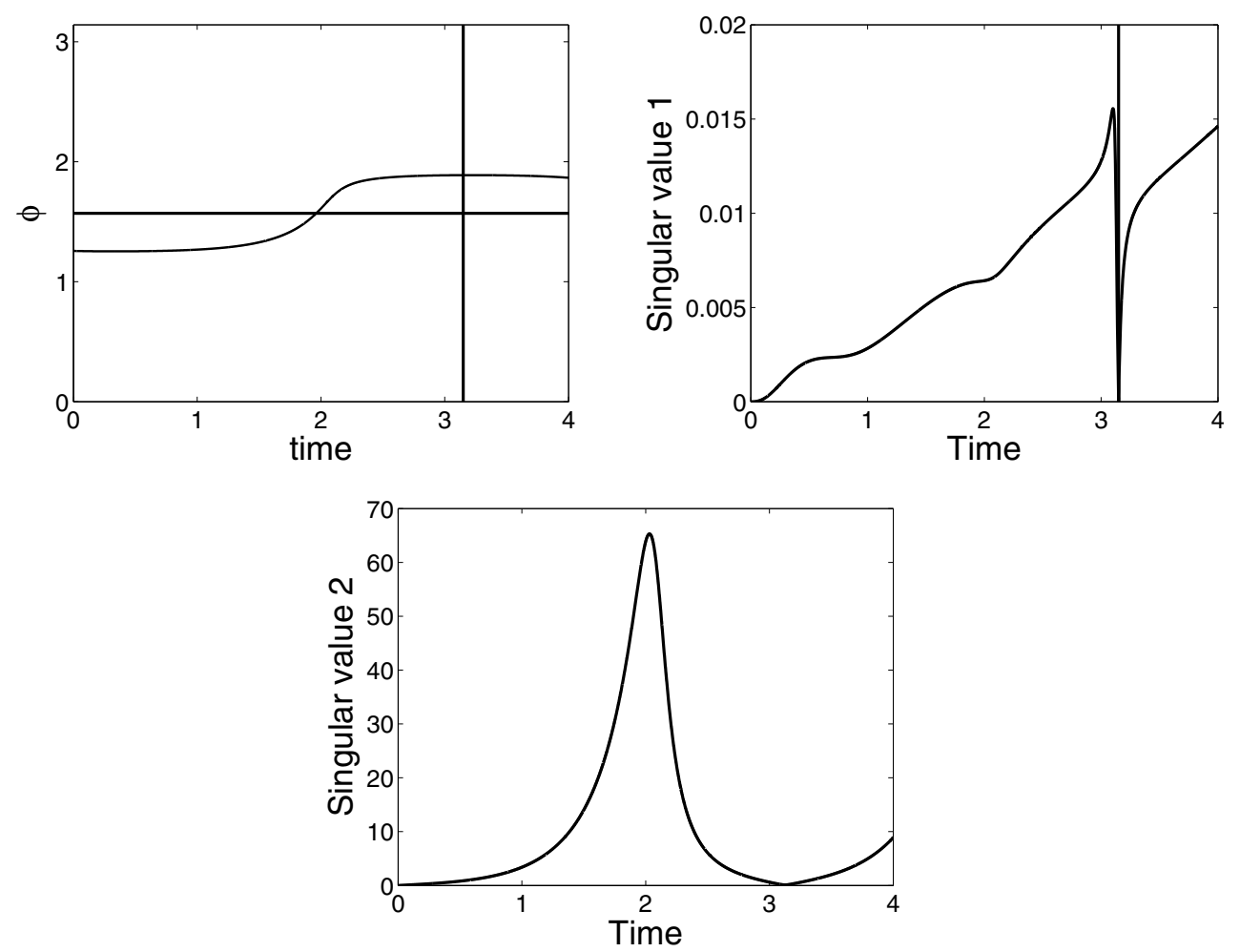

Figure 4. Same as Figure 3 but for $p_{\phi}(0)=2.637$. The vertical solid line indicates the position of the first conjugate point along the extremal trajectory.

- In the generic case $\gamma_{-} \neq 0$ conjugate points are observed in case a) as illustrated in Figure 5 whereas there is no conjugate point in case b) as can be seen in Figure 6.

\subsection{The continuation method}

The continuation method was both implemented in a simple numerical framework using MatLab routines (discretization of the parameter interval which can be normalized to [0,1], and at each step the Newton routine was used to compute the root) and by using a smooth approach. In this second case, a unique shooting is necessary to initialize the continuation method.

For geometric reasons the continuation method is splitted into two parts.

- Continuation method for the reduced problem: the final purity is not controlled and the transversality condition $p_{\rho}\left(t_{f}\right)=0$ at the final time $t_{f}$ is added in the shooting equation. Observe that for this modified shooting problem the rank condition is equivalent to the non-existence of focal points (see [12]).

- Continuation method for the full problem. In this case, we have to check that the final purity belongs to the accessible set for every value of the dissipation parameters.

We present two numerical examples illustrating these two situations. The smooth and the discrete continuation approaches have been implemented in each case. We denote respectively by $\left(p_{\rho f}=0, \phi_{f}, \theta_{f}\right)$ and $\left(\rho_{f}, \phi_{f}, \theta_{f}\right)$ the target states of the control in the first and second cases. In the first case where the final purity is not fixed, we consider the homotopy method with respect to the parameter $\Gamma$ and in the second case a continuation with respect to $\gamma_{-}$. Knowing the starting point of the continuation method $\left(p_{\rho}(0), p_{\phi}(0), t_{f}\right)$, we use the two continuation algorithms to determine new triplets $\left(p_{\rho}(0), p_{\phi}(0), t_{f}\right)$ such that the system reaches the same target 

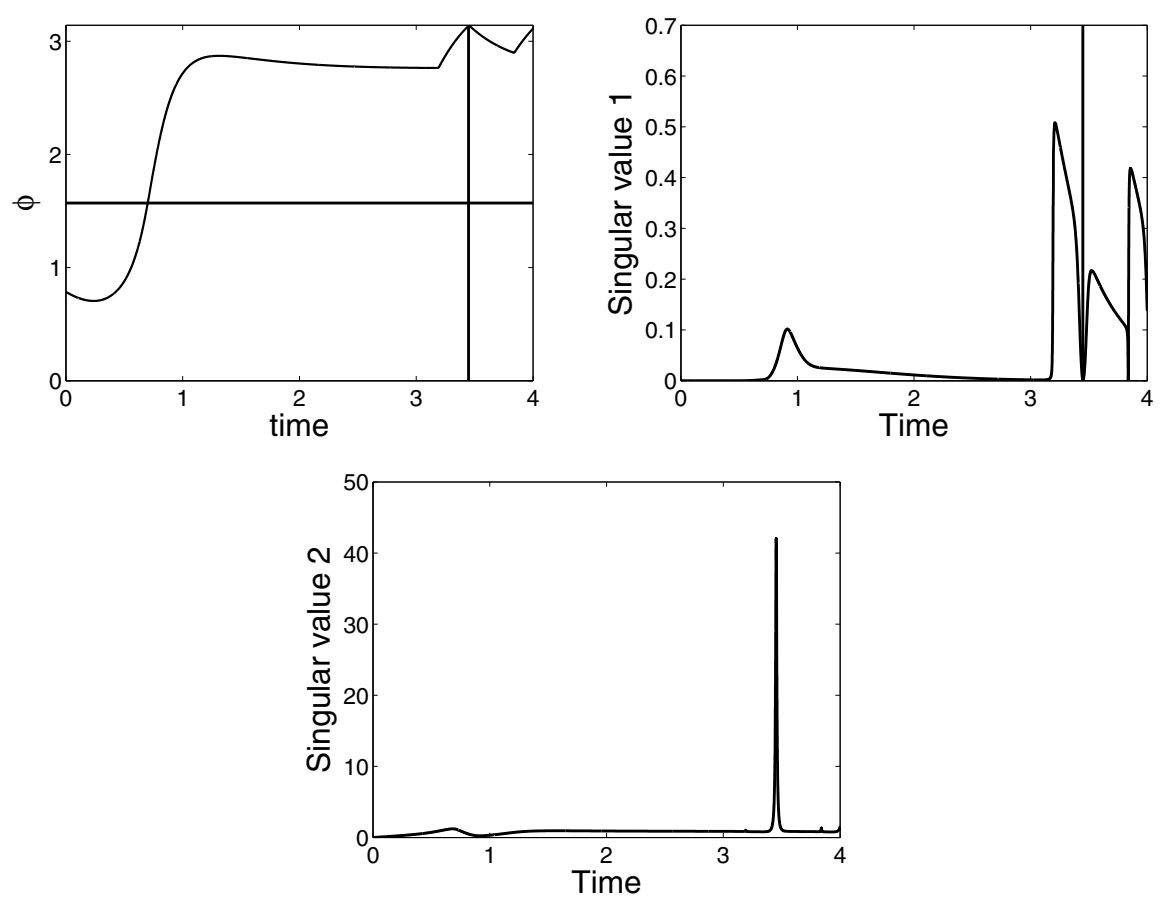

Figure 5. Same as Figure 4 but for $\Gamma=2.5, \gamma_{+}=2, \gamma_{-}=-0.5, \phi(0)=\pi / 4, p_{\rho}(0)=0.1$, $p_{\theta}=2$ and $p_{\phi}(0)=-2$.
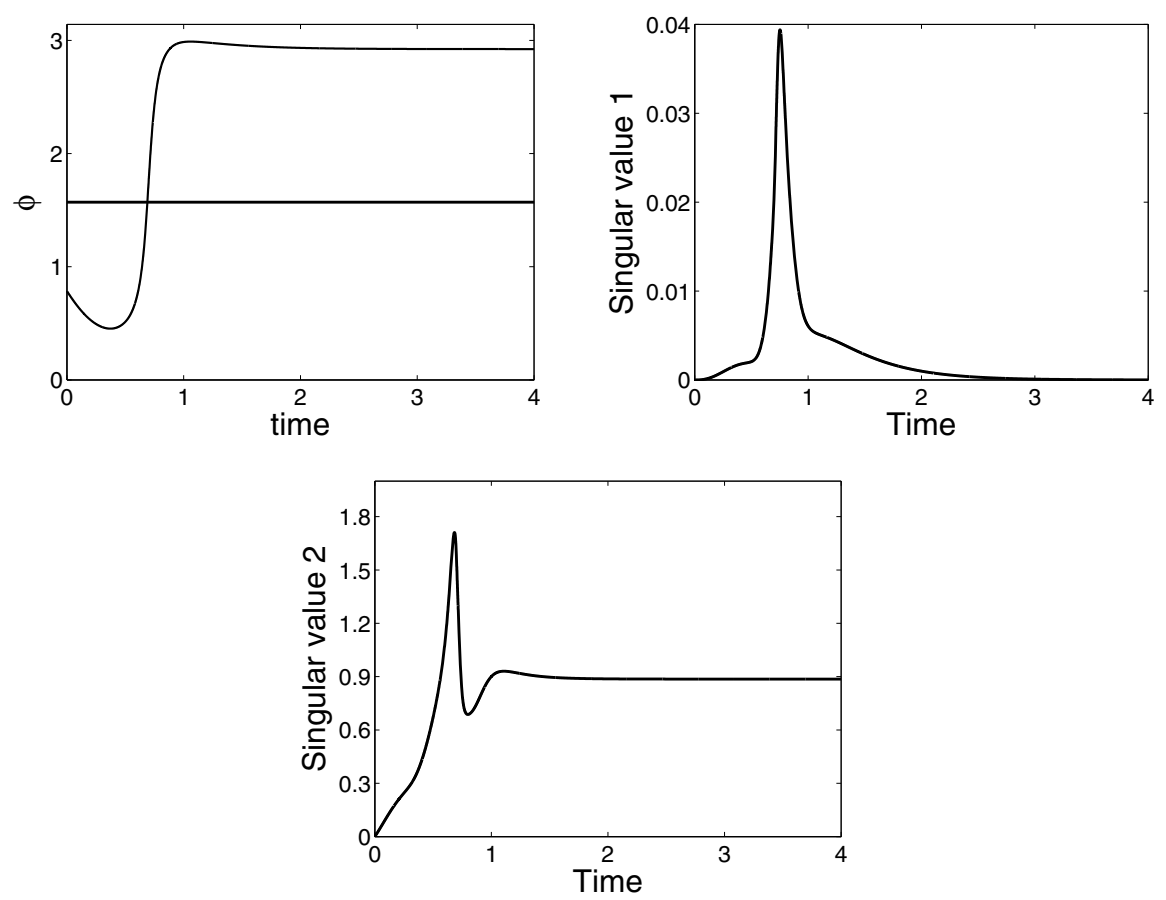

Figure 6 . Same as Figure 5 but for $\Gamma=4.5$. There is no conjugate point. 

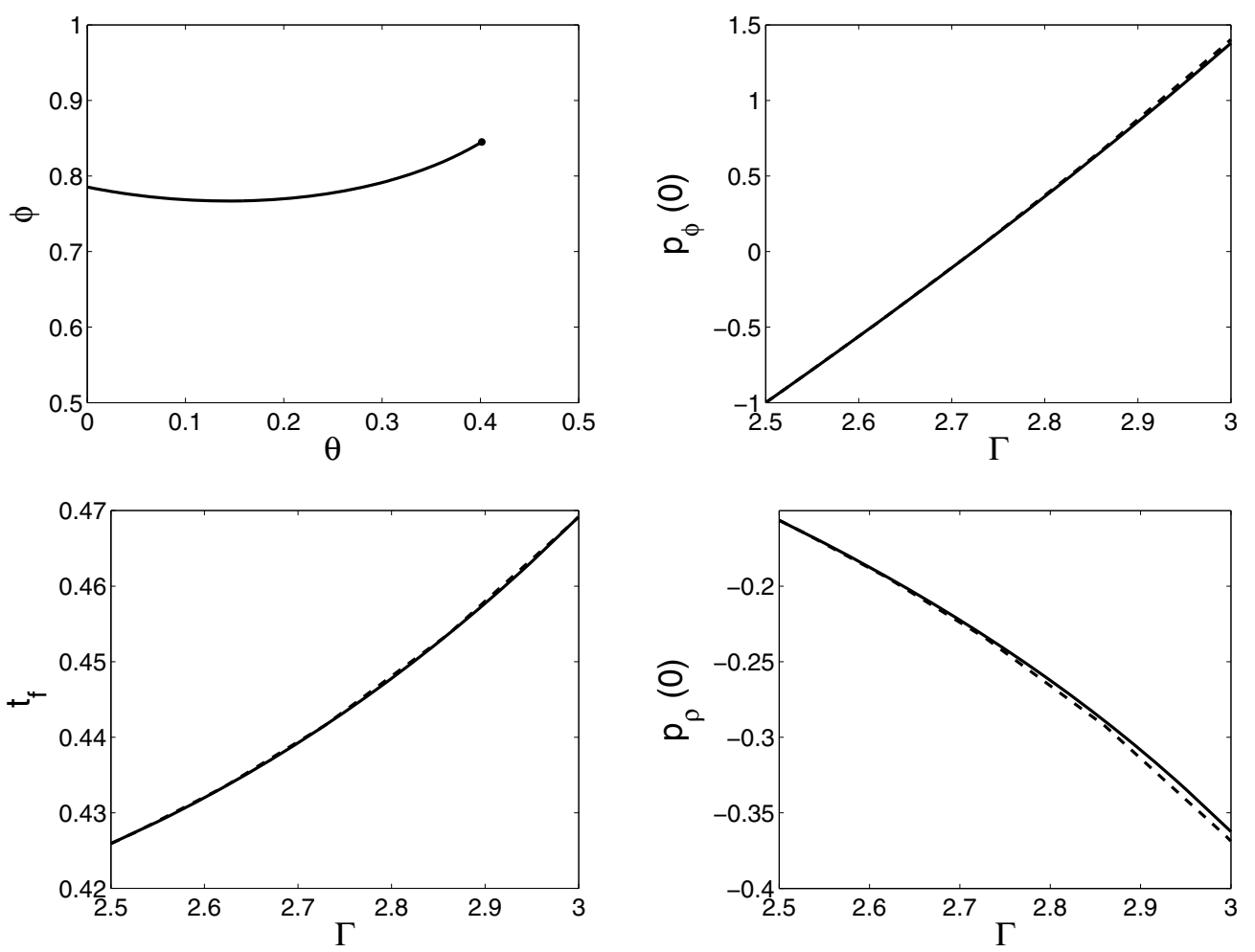

Figure 7. Continuation with respect to $\Gamma$. The parameters of the initial point are $\Gamma=2.5$, $\gamma_{+}=2, \gamma_{-}=-0.1, \phi(0)=\pi / 4, p_{\rho}(0)=-0.1564, p_{\theta}=8$ and $p_{\phi}(0)=-1$. The initial time is $t_{f}=0.4259$. The target state corresponds to $\phi_{f}=0.845096, \theta_{f}=0.04015590$ and $p_{\rho f}=0$. Three figures represent the evolution of the adjoint states $p_{\rho}(0)$ and $p_{\phi}(0)$ and of the control duration $t_{f}$ as a function of $\Gamma$ for the Newton-type continuation (dashed line) and the smoothtype one (solid line). The last figure displays the projection of the extremal trajectory solution of the continuation problem onto the Bloch sphere for $\Gamma=2.5$ and $\gamma_{+}=2$.

state when the dissipation parameters vary. The adjoint state $p_{\theta}$ is kept fixed in the computation, while the value of the Hamiltonian, which remains positive, changes at each step. Figures 7 and 9 represent the different results we have obtained. In each case the control duration is chosen small enough to be before the first conjugate point. Note the smooth evolution of the three parameters $p_{\phi}(0), p_{\rho}(0)$ and $t_{f}$ when the continuation parameters vary. The results of the two methods are very close to each other. The step size of the discrete approach is respectively taken to be 0.05 and 0.01 in the first and second cases. With this step size which is not representative of our control problem (larger step size can be chosen), the Newton algorithm does not encounter any problem to converge. The shooting equation is solved with an accuracy better than $10^{-12}$ in the two continuation methods.

In the second case, when we consider a final condition on the radial coordinate, some problems of accessibility have been encountered. As a preliminary study, we have therefore to check that the target state belongs to the accessibility set for every value of the dissipation parameters used in the continuation method. When this condition is satisfied, we have observed no problem of convergence for the continuation method. This condition is more difficult to fulfill for long control durations. Figure 11 illustrates the constraint due to the accessibility set in the second case. For given values of dissipation parameters and of $p_{\rho}$, we have determined the values of $p_{\phi}(0)$ and $t_{f}$ allowing to reach the point of coordinates $\left(\phi_{f}, \theta_{f}\right)$. We have then plotted the corresponding 


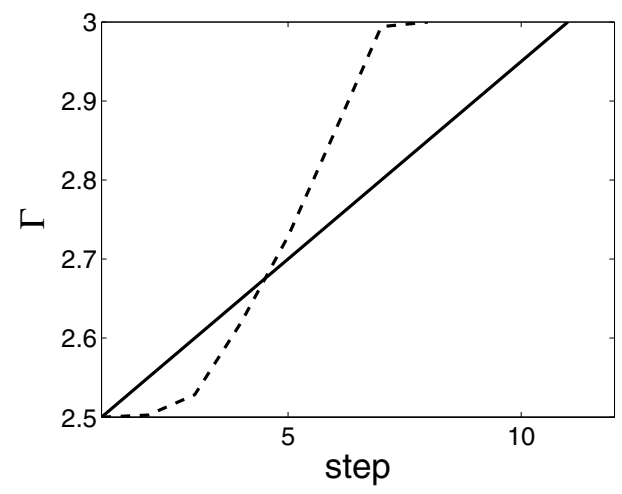

FIGURE 8. Evolution of the continuation parameter $\Gamma$ as a function of the number of steps for the discrete method (solid line) and the smooth one (dashed line).
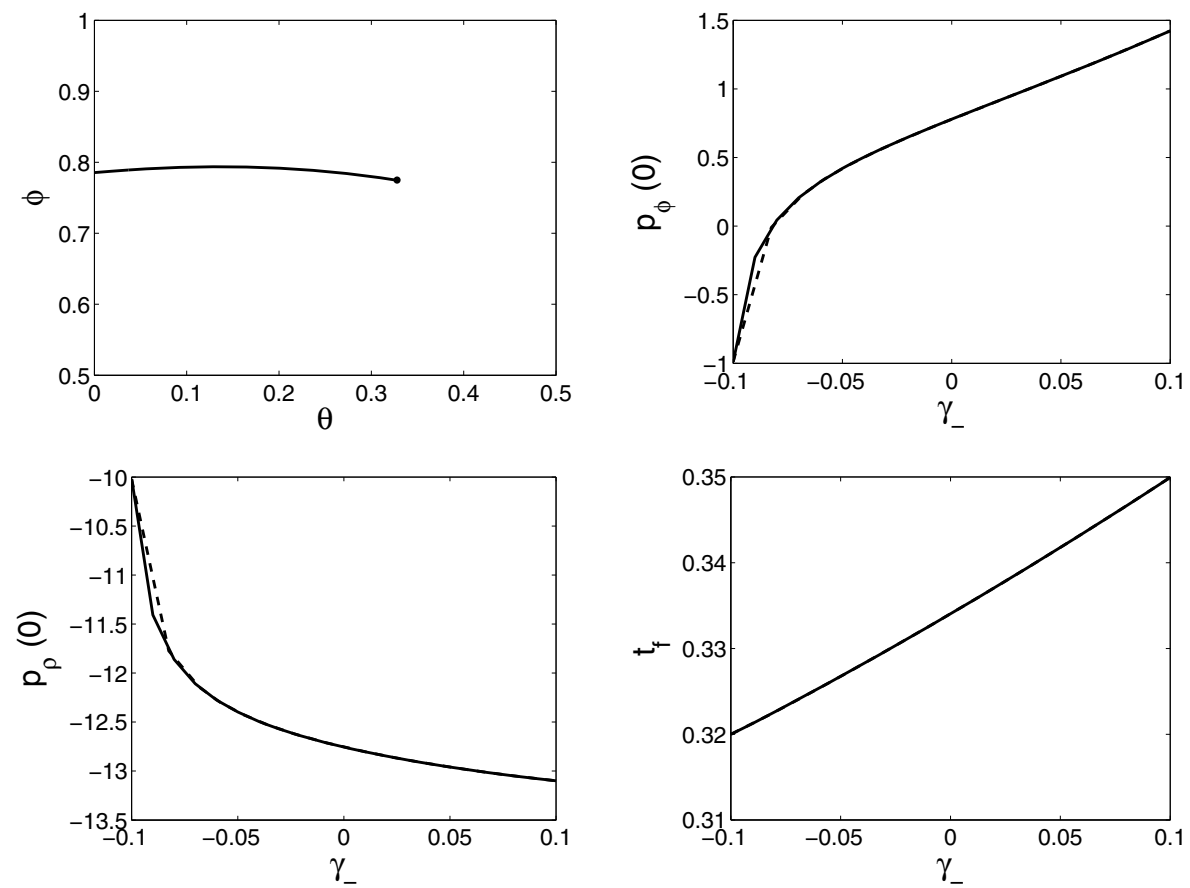

Figure 9. Same as Figure 7 but for a continuation with respect to $\gamma_{-}$. The parameters of the initial point are $\Gamma=2.5, \gamma_{+}=2, \gamma_{-}=-0.1, \phi(0)=\pi / 4, p_{\rho}(0)=-10.0208, p_{\theta}=8$ and $p_{\phi}(0)=-1$. The initial time is $t_{f}=0.32$. The target state corresponds to $\rho_{f}=0.47182$, $\phi_{f}=0.77468$ and $\theta_{f}=0.32774$.

radial coordinate $\rho_{f}$ as a function of $p_{\rho}$. Note that the diagram is symmetric with respect to the vertical axis $p_{\rho}=0$ and has roughly the same form up to vertical translations for different values of the dissipation parameters. Figure 11 shows the values of the radial coordinate $\rho_{f}$ that can be reached when $\phi_{f}$ and $\theta_{f}$ are fixed. Using such a diagram for different dissipation parameters, one can check that the point $\left(\rho_{f}, \phi_{f}, \theta_{f}\right)$ belongs to the accessible set. Figures 8 and 10 represent the evolution of the continuation parameters as a function of 


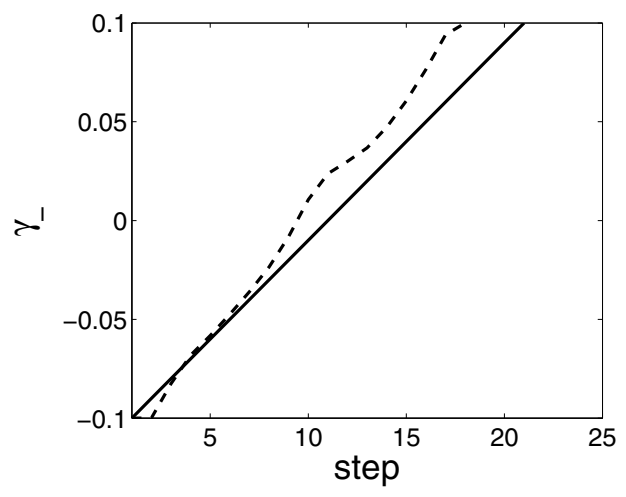

Figure 10. Same as Figure 8 but for the continuation parameter $\gamma_{-}$.

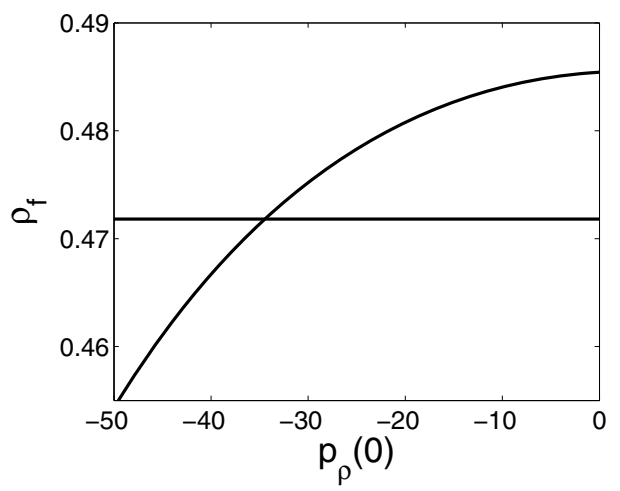

Figure 11. Plot of $\rho_{f}$ as a function of $p_{\rho}(0)$ for $\Gamma=2.5, \gamma_{+}=2$ and $\gamma_{-}=0.1$. Other parameters are taken to be $\phi(0)=\pi / 4, p_{\theta}=8, \phi_{f}=0.77468$ and $\theta_{f}=0.32774$. The equation of the horizontal line is $\rho_{f}=0.47182$, which corresponds to the target state.

the number of steps. The step size of the Newton algorithm has been chosen so that the number of steps of the two approaches be roughly the same. Note the non-trivial behavior of the continuation parameter in the second case.

\section{REFERENCES}

[1] A.A. Agrachev and Y.L. Sachkov, Control theory from the geometric viewpoint, Encyclopaedia of Mathematical Sciences 87, Control Theory and Optimization II. Springer-Verlag, Berlin, Germany (2004).

[2] E.L. Allgower and K.G. Georg, Introduction to numerical continuation methods, SIAM Classics in Applied Maths 45. Society for Industrial and Applied Mathematics, Philadelphia, USA (2003).

[3] D. Bao, C. Robles and Z. Shen, Zermelo navigation on Riemannian manifolds. J. Differential Geom. 66 (2004) $377-435$.

[4] A.G. Bliss, Lectures on the Calculus of Variations. University of Chicago Press, Chicago, USA (1946).

[5] B. Bonnard and I. Kupka, Théorie des singularités de l'application entrée/sortie et optimalité des trajectoires singulières dans le problème du temps minimal [Theory of the singularities of the input/output mapping and optimality of singular trajectories in the minimal-time problem]. Forum Math. 5 (1993) 111-159.

[6] B. Bonnard and D. Sugny, Time-minimal control of dissipative two-level quantum systems: the integrable case. SIAM J. Control Optim. 48 (2009) 1289-1308.

[7] B. Bonnard and D. Sugny, Geometric optimal control and two-level dissipative quantum systems. Control Cybern. (to appear). 
[8] B. Bonnard, L. Faubourg and E. Trélat, Mécanique céleste et contrôle des véhicules spatiaux. Springer, Berlin, Germany (2005).

[9] B. Bonnard, R. Dujol and J.-B. Caillau, Smooth approximations of single-input controlled Keplerian trajectories: homotopies and averaging, in Taming heterogeneity and complexity of embedded control, Proceedings of the Joint CTS-HYCON Workshop on Nonlinear and Hybrid Control, Paris, France (2006) 73-95.

[10] B. Bonnard, J.-B. Caillau and E. Trélat, Second order optimality conditions in the smooth case and applications in optimal control. ESAIM: COCV 13 (2007) 207-236.

[11] B. Bonnard, J.-B. Caillau, R. Sinclair and M. Tanaka, Conjugate and cut loci of a two-sphere of revolution with application to optimal control. Ann. Inst. H. Poincaré Anal. Non Linéaire 26 (2009) 1081-1098.

[12] B. Bonnard, M. Chyba and D. Sugny, Time-minimal control of dissipative two-level quantum systems: the generic case. IEEE Trans. Automat. Contr. 54 (2009) 2595-2610.

[13] B. Bonnard, O. Cots, N. Shcherbakova and D. Sugny, The energy minimization problem for two-level dissipative quantum systems. J. Math. Phys. (to appear).

[14] U. Boscain and P. Mason, Time minimal trajectories for a spin 1/2 particle in a magnetic field. J. Math. Phys. 47 (2006) 062101.

[15] H.-P. Breuer and F. Petruccione, The theory of open quantum systems. Oxford University Press, London, UK (2002).

[16] D. D'Alessandro, Introduction to quantum control and dynamics, Applied Mathematics and Nonlinear Science Series. Chapman \& Hall/CRC, Boca Raton, USA (2008).

[17] M.P. do Carmo, Riemannian geometry. Birkhauser, Boston, USA (1992).

[18] R. Dujol, Contribution du contrôle orbital des transferts mono-entrée en mécanique spatiale. Ph.D. Thesis, ENSEEIHT-INP, France (2006).

[19] J. Gergaud and T. Haberkorn, Homotopy method for minimum consumption orbit transfer problem. ESAIM: COCV 12 (2006) $294-310$.

[20] T. Haberkhorn, Transfert orbital avec minimisation de la consommation : résolution par homotopie différentielle. Ph.D. Thesis, ENSEEIHT-INP, France (2004).

[21] N. Khaneja, R. Brockett and S.J. Glaser, Time optimal control of spin systems. Phys. Rev. A. 63 (2001) 032308.

[22] N. Khaneja, S.J. Glaser and R. Brockett, Sub-Riemannian geometry and time optimal control of three spin systems: quantum gates and coherence transfer. Phys. Rev. A (3) 65 (2002) 032301.

[23] D.F. Lawden, Elliptic functions and applications. Springer Verlag, New York, USA (1989).

[24] H. Maurer and H.J. Oberle, Second order sufficient conditions for optimal control problems with free final time: the Riccati approach. SIAM J. Control Optim. 41 (2002) 380-403.

[25] L.S. Pontryagin, V.G. Boltyanskii, R.V. Gamkrelidze and E.F. Mishchenko, The mathematical theory of optimal processes. L.W. Neustadt Interscience Publishers, John Wiley \& Sons, Inc., New York-London (1962).

[26] A. Sarychev, The index of the second variation of a control system. Math. Sbornik 41 (1982) $383-401$.

[27] T. Schulte-Herbrüggen, A.K. Spörl, R. Marx, N. Khaneja, J.M. Myers, A.F. Fahmy and S.J. Glaser, Quantum computing implemented via optimal control: Theory and application to spin and pseudo-spin systems, in Lectures on quantum information, D. Bruß and G. Leuchs Eds., Wiley-VCH (2006) 481.

[28] T. Vieillard, F. Chaussard, D. Sugny, B. Lavorel and O. Faucher, Field-free molecular alignment of CO 2 mixtures in presence of collisional relaxation. J. Raman Spec. 39 (2008) 694.

[29] R. Wu, A. Pechen, H. Rabitz, M. Hsieh and B. Tsou, Control landscapes for observable preparation with open quantum systems. J. Math. Phys. 49 (2008) 022108. 